Aubram, D.

\title{
Homogeneous equilibrium model for geomechanical multi-material flow with compressible constituents
}

Journal article | Accepted manuscript (Postprint)

This version is available at https://doi.org/10.14279/depositonce-7251

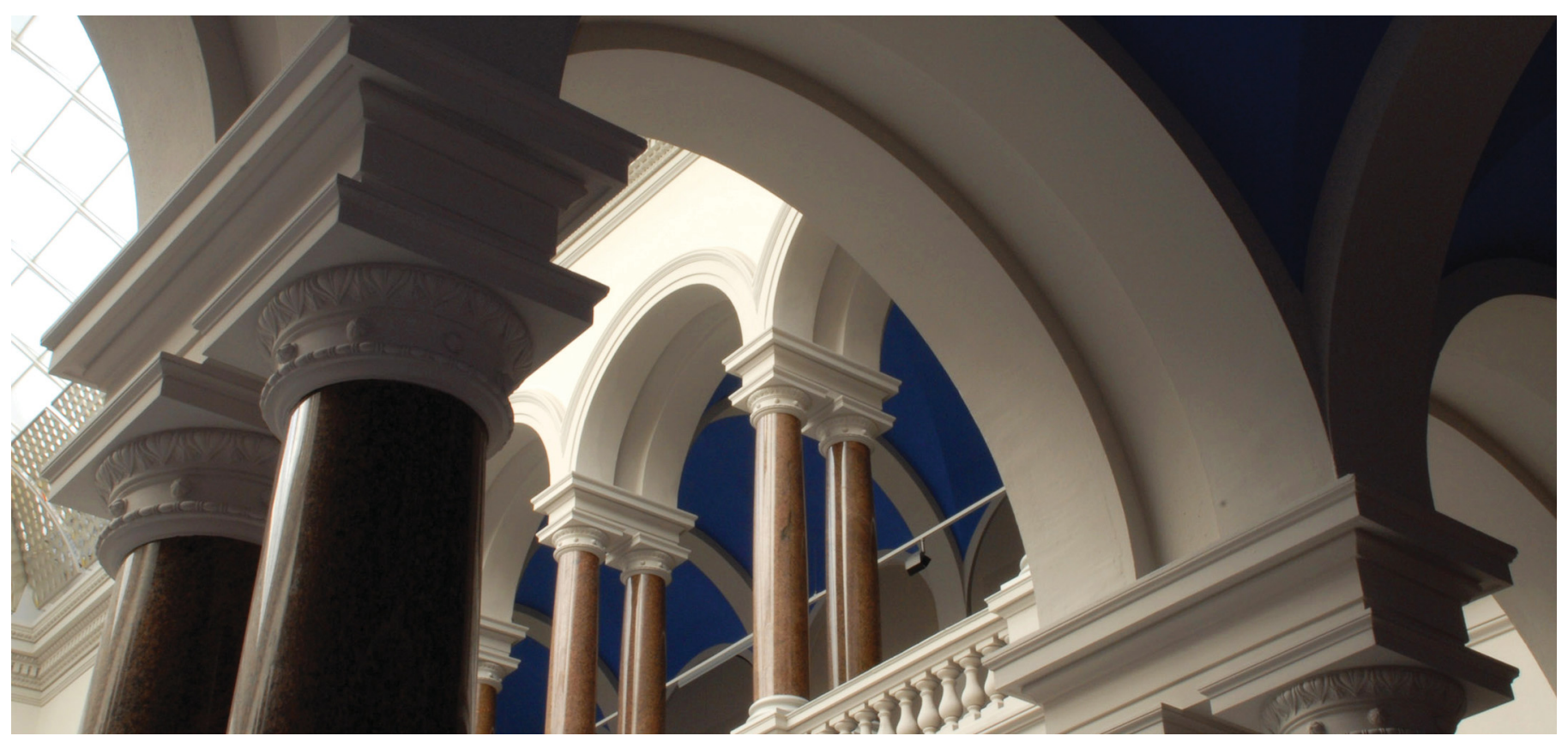

Aubram, D. (2016). Homogeneous equilibrium model for geomechanical multi-material flow with compressible constituents. Journal of Non-Newtonian Fluid Mechanics, 232, 88-101. https://doi.org/10.1016/j.jnnfm.2016.04.001 


\title{
Homogeneous equilibrium model for geomechanical multi-material flow with compressible constituents
}

\author{
D. Aubram* \\ Chair of Soil Mechanics and Geotechnical Engineering, Berlin University of Technology \\ (TU Berlin), Secr. TIB1-B7, Gustav-Meyer-Allee 25, D-13355 Berlin, Germany
}

\begin{abstract}
Multi-material flow generally describes a situation where several distinct materials separated by sharp material interfaces undergo large deformations. In order to model such flow situations in the context of geomechanics and geotechnical engineering, a theoretical framework is presented which introduces a possible two-phase coupled saturated granular material behavior among the different materials. This is achieved by extending the technique of local volume averaging to a hierarchy of three spatial scales, based on a product of two indicator functions. A homogeneous equilibrium mixture model is subsequently derived for an example flow consisting of bulk solid, bulk fluid, and undrained granular material with compressible constituents. The closure relations are provided at the macroscale, including those describing granular behavior covering the full frictional-collisional flow regime and bulk material volume fraction evolution. The paper discusses the advantages and restrictions of the proposed mixture model and addresses its application and full-scale numerical implementation.
\end{abstract}

*Tel.: +49 (0)30 31472341; Fax: +49 (0)30 31472343.

E-mail address: daniel.aubram@tu-berlin.de

Copyright (C) 2016. This manuscript version is made available through CC-BY-NC-ND 4.0 license http://creativecommons.org/licenses/by-nc-nd/4.0

This is the author's version of a work that was accepted for publication. A definitive version of this article is published in: D. Aubram, Homogeneous equilibrium model for geomechanical multi-material flow with compressible constituents, Journal of Non-Newtonian Fluid Mechanics (2016), http://dx.doi.org/10.1016/j.jnnfm.2016.04.001 
Keywords: multi-material flow, large deformations, mixture, suspension, sand, granular material, volume averaging, closure relation, homogeneous equilibrium

\section{Introduction}

Multi-material flow describes a situation where several pure, physically distinct materials (solids, liquids, gases) interact and one or more of these materials undergo large deformations — void, representing empty space or atmosphere, is generally considered as material. In contrast to multi-phase or multi-fluid flow $[1,2,3,4,5]$, the main characteristics of multi-material flow are the evolution of large-scale material interfaces, including the generation of new free surfaces or the coalescence of existing surfaces, as well as the presence of material strength and compressibility. Moreover, in many situations mass transfer between the materials is of secondary interest, and momentum and pressure relaxation can be assumed infinitely fast, resulting in velocity and pressure fields common to all materials of the flow.

The notion of multi-material flow has emerged along with the development of efficient numerical simulation techniques to analyze detonation and impact problems, the dynamics of bubbles and droplets, material processing and manufacturing, or astrophysical events $[6,7,8]$. The most attractive approaches use Eulerian or arbitrary Lagrangian-Eulerian (ALE) descriptions allowing interfaces and free surfaces to flow through the computational mesh $[9,10,11,12,13,14]$. Mesh cells cut by interfaces (multi-material cells) necessarily arise which contain a heterogeneous mixture of two or more materials. In order to solve the same equations with the same numerical method, the 
heterogeneous mixture is represented as an effective single-phase material or homogenized mixture by using physically based mixing rules [15, 16, 17].

The research presented in this paper addresses multi-material flow situations encountered in geomechanics and geotechnical engineering. Examples are natural hazards like landslides [18, 19], avalanches and debris flows [20, 21, 22], liquefaction-induced soil failure [23, 24], and elementary installation processes like digging, injection, mixing, displacement, or penetration $[25,26,27,28,29]$. Schematic views are shown in Fig. 1. Besides the characteristics common to all multi-material flows, the aforementioned problems involve a complex coupled behavior of the dense grain-fluid mixture representing the soil or debris material as well as a hierarchy of distinct spatial scales [30]. While certain aspects of such geomechanical multi-material flows can be considered as well understood, a fully-fledged flow model that is able to predict a time history of the material states for arbitrary compositions and configurations of the mixture is yet missing.

The paper is concerned with the development of a macroscopic mechanical theory for compressible multi-material flow involving a hierarchy of three scales. We proceed from the premise not to describe small-scale details in the flow field, but rather large-scale motions and interactions of bulk materials. By assuming homogeneous equilibrium between all constituents of the flow and by making use of local volume averaging, we provide a closed set of equations that holds at each spatial point and at all interfaces. In contrast to two-scale theories which consider the motion of multiple single-phase materials or that of a single multi-phase mixture, our three-scale approach incorporates the evolution of interfaces as well as two-phase phenomena as- 
(a)
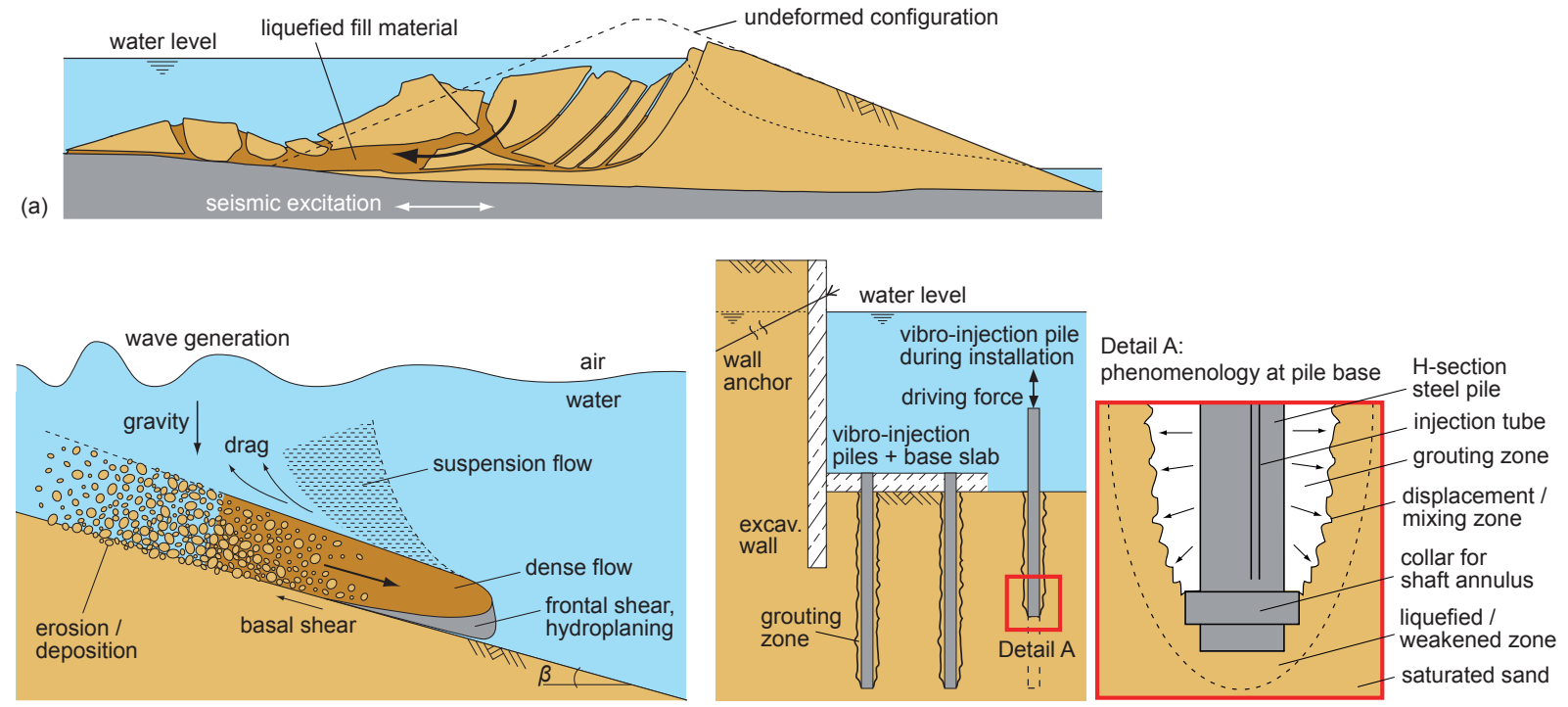

(b)

(c)

Figure 1: Schematic of complex geomechanical multi-material flow situations. (a) Liquefaction-induced failure of an earth-fill dam under seismic excitation; in accordance with [23]. (b) Submarine landslide; in accordance with [18]. (c) Installation of vibroinjection piles to tie back the base slab of a deep excavation; in accordance with [28]. Reprint from [30, p. 189] with permission of Springer

sociated with saturated granular materials. For simplicity, we ignore any thermodynamical issue and do not specify any boundary conditions characterizing a particular multi-material flow. Applications and comparisons with experiments or numerical simulations are not considered.

The structure of the remaining text is as follows. Section 2 derives macroscopic balance principles for a mixture of multiple materials consisting of multiple phases by making use of local volume averaging. The averaging procedure involves some new aspects regarding the special flow structure. The description of fluid-saturated granular material is addressed in Section 3. We take into account compressible constituents as well as rate-independent 
frictional and rate-dependent viscous (collisional) contributions to the stress tensor. In Section 4, a reduced mixture model is developed for a geomechanical multi-material example flow by assuming homogeneous equilibrium of pressure and velocity. Closure relations are specified in order to render the reduced model well posed. Section 5 discusses the advantages and restrictions of the homogeneous equilibrium mixture model and addresses its application and full-scale numerical implementation. The paper closes with concluding remarks and outlook in Section 6.

\section{Averaged equations for three-scale mixture}

\subsection{Averaging procedure}

The flow of interest is a three-scale (micro, meso, macro) system consisting of a bulk solid $(\mathrm{S})$, a bulk fluid $(\mathrm{F})$, and a composite material representing fluid-saturated granular material $(\mathrm{G})$. The granular material is an immiscible mixture consisting of a solid phase (s) and fluid phase (f). Void is considered as a particular fluid. The situation is illustrated in Fig. 2.

The granular material is constituted by an assembly of solid grains, whose

typical diameter defines the microscale of the problem, $l_{\text {micro }}$ (Fig. 2 below). The grain assembly can be represented by a continuum at the mesoscale $l_{\text {meso }}$, separated from the bulk solid and bulk fluid by sharp interfaces. Finally, we assume that the multi-material flow has a representative volume element (RVE) with characteristic length $l_{\text {macro }}$ referred to as the macroscale (Fig. 2 above). At the macroscale, the immiscible mixture of mesoscale continua can be equivalently modeled as an effective single-phase material, i.e. homogeneous mixture. 


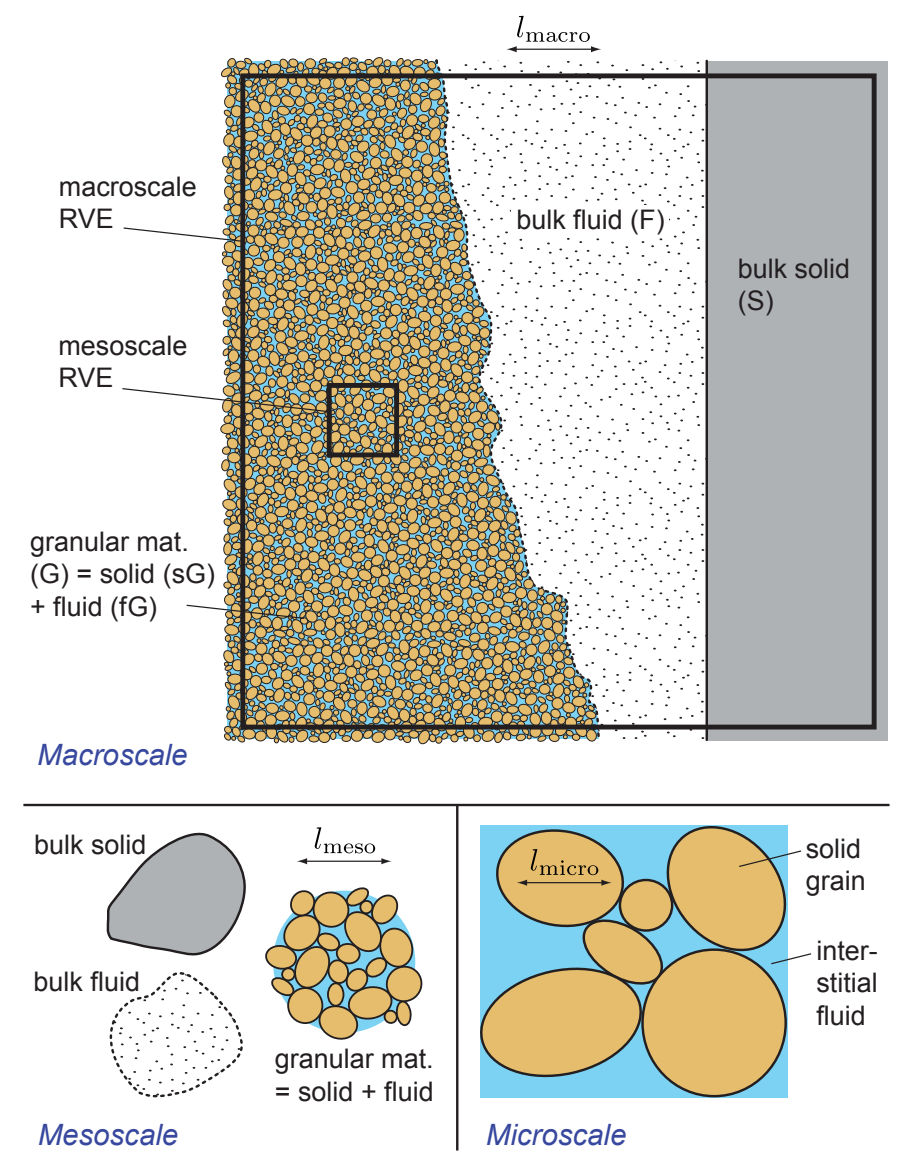

Figure 2: Three spatial scales in a particular geomechanical multi-material flow. Reprint from $[30$, p. 195] with permission of Springer

To keep our theory as general as possible, each material $k \in\{\mathrm{S}, \mathrm{F}, \mathrm{G}\} \stackrel{\text { def }}{=}$ $\{1, \ldots, M\}$ is initially viewed as containing the same phases $\alpha \in\{\mathrm{s}, \mathrm{f}\} \stackrel{\text { def }}{=}$ $\{1, \ldots, N\}$, even though the fractional volume of one phase in a particular material might be zero. A particular phase $\alpha$ in a particular material $k$ represents an individual, chemically-independent constituent of the flow and will be denoted by $\alpha k$. For the particular flow under consideration we write $\mathrm{sS} \equiv \mathrm{S}$ and $\mathrm{fF} \equiv \mathrm{F}$ such that $\alpha k \in\{\mathrm{S}, \mathrm{F}, \mathrm{sG}, \mathrm{fG}\}$. The complete 
nomenclature is found at the end of this paper.

The flow takes place in a domain $\mathcal{D} \subset \mathbb{R}^{3}$, and the subregions in $\mathcal{D}$ instantaneously occupied by the $k$-material and the $\alpha$-phase at time $t \in[0, T]$ are denoted by $\mathcal{M}^{k}$ and $\mathcal{P}^{\alpha}$, respectively. We require

$$
\mathcal{D}=\bigcup_{\alpha} \mathcal{P}^{\alpha}=\bigcup_{k} \mathcal{M}^{k}
$$

The domain of the $\alpha$-phase in the $k$-material is given by the intersection $\mathcal{P}^{\alpha} \cap \mathcal{M}^{k}$. The intersection of each two phases and each two materials, based on the assumptions above, is either empty or the shared interface.

Let $\chi^{k}$ be the material indicator function on $\mathcal{M}^{k} \subset \mathcal{D}$ and $\chi^{\alpha}$ the phase indicator function on $\mathcal{P}^{\alpha} \subset \mathcal{D}$, with $\chi^{k}, \chi^{\alpha}: \mathcal{D} \times[0, T] \rightarrow\{0,1\}$. The indicator function which picks out the $\alpha$-phase domain of the $k$-material domain is the product $\chi^{\alpha} \chi^{k} \stackrel{\text { def }}{=} \chi^{\alpha k}$ :

$$
\chi^{\alpha k}(x, t)= \begin{cases}1 & \text { if } x \in\left(\mathcal{P}^{\alpha} \cap \mathcal{M}^{k}\right) \text { at time } t, \\ 0 & \text { if } x \in \mathcal{D} \backslash\left(\mathcal{P}^{\alpha} \cap \mathcal{M}^{k}\right) \text { at time } t .\end{cases}
$$

This indicator function is unique to our theory and covers arbitrary flow compositions bounded between the classical cases of mixtures of single-phase materials $\left(\chi^{\alpha}=1\right)$ and mixtures represented by a single multiphase material $\left(\chi^{k}=1\right)$.

We employ hybrid mixture theory $[31,32,33,34,35]$ to upscale information from the microscale to the macroscale; this and other types of approaches are reviewed in $[36,37]$. The basic idea in hybrid mixture theory is to apply local volume averaging $[38,39,5]$ to the small scale balance equations and to make the constitutive assumptions needed for closure at the large scale, i.e. for the averaged balance equations. Consider a macroscale RVE 
of the flow, $\mathcal{V}(x) \subset \mathbb{R}^{3}$, at all points $x \in \mathcal{D}$. At each instant $t$, the RVE intersects with the current configuration of materials and phases as well as with their boundaries. For example, the $k$-material occupies the subregion $\mathcal{M}^{k} \cap \mathcal{V} \stackrel{\text { def }}{=} \mathcal{V}^{k}$ of the RVE, and $\left(\mathcal{P}^{\alpha} \cap \mathcal{M}^{k}\right) \cap \mathcal{V} \stackrel{\text { def }}{=} \mathcal{V}^{\alpha k}$ is the subregion occupied by the $\alpha$-phase of the $k$-material. Note that $\mathcal{V}=\bigcup_{k} \mathcal{V}^{k}=\bigcup_{k} \bigcup_{\alpha} \mathcal{V}^{\alpha k}$ by requirement (1).

The $\mathcal{V}$-average of an arbitrary time-dependent spatial microscopic field $q(x, t)$ is defined through

$$
\langle q\rangle(x, t) \stackrel{\text { def }}{=} \frac{1}{V} \int_{\mathcal{V}} q(x+\boldsymbol{y}, t) \mathrm{d} v
$$

for all $x \in \mathcal{D}$ and $t \in[0, T]$, where $\mathrm{d} v$ is the volume density on $\mathbb{R}^{3}, V \stackrel{\text { def }}{=}$ $\int_{\mathcal{V}} 1 \mathrm{~d} v=$ const is the volume measure of $\mathcal{V}$, and $\boldsymbol{y} \in \mathcal{V}$ is a vector. In particular, we the define volume fractions

$$
f^{k} \stackrel{\text { def }}{=}\left\langle\chi^{k}\right\rangle=\frac{V^{k}}{V} \quad \text { and } \quad \pi^{\alpha k} \stackrel{\text { def }}{=} \frac{1}{f^{k}}\left\langle\chi^{\alpha k}\right\rangle=\frac{V^{\alpha k}}{V^{k}},
$$

where $V^{k} \stackrel{\text { def }}{=} \int_{\mathcal{V}^{k}} 1 \mathrm{~d} v=\int_{\mathcal{V}} \chi^{k} \mathrm{~d} v$ and $V^{\alpha k} \stackrel{\text { def }}{=} \int_{\mathcal{V}^{\alpha k}} 1 \mathrm{~d} v=\int_{\mathcal{V}} \chi^{\alpha k} \mathrm{~d} v$. While $f^{k}$ is the volume fraction of the $k$-material with respect to the RVE, $\pi^{\alpha k}$ represents the macroscale volume fraction of the $\alpha$-phase intrinsic to the $k$ material, with $f^{k}, \pi^{\alpha k} \in[0,1]$. Since phase or material overlaps are precluded, one has

$$
\sum_{k} f^{k}=1 \quad \text { and } \quad \sum_{\alpha} \pi^{\alpha k}=1
$$

for all $k \in\{1, \ldots, M\}$.

For microscopic $q$ defined per unit volume, the corresponding field of the mixture at the macroscale,

$$
\langle q\rangle=\sum_{k} f^{k} q^{k}=\sum_{k} \sum_{\alpha} f^{k} \pi^{\alpha k} q^{\alpha k}
$$


with

$$
q^{\alpha k} \stackrel{\text { def }}{=} \frac{\left\langle\chi^{\alpha k} q\right\rangle}{f^{k} \pi^{\alpha k}},
$$

follows immediately from (3) and (5). The superscribed $\alpha k$ denotes macroscopic (i.e. $\mathcal{V}$-averaged) fields related to the $\alpha$-phase in the $k$-material. For example, if $q=\rho$ is the microscopic spatial mass density, then the intrinsic or true mass density $\rho^{\alpha k}$ represents the mass of the constituent $\alpha k$ per unit volume of that constituent, $\pi^{\alpha k} \rho^{\alpha k}$ is the mass of the constituent $\alpha k$ per unit

volume of the $k$-material, and $f^{k} \pi^{\alpha k} \rho^{\alpha k}$ denotes its mass per unit volume of the mixture.

\subsection{Balance equations}

On the microscale all constituents of the mixture are regarded as continua, governed by the equations of continuum mechanics [40, 41, 42]. The balance equations of the problems under consideration are conservation of mass and balance of momentum, in conjunction with the interface jump conditions. In the microscopic balance equations, a single spatial point is viewed as being instantaneously occupied by a single constituent. For simplicity, we do not take care of any thermodynamical issue. Moreover, we do not attribute any mechanical properties to the interfaces, i.e. neither interfacial mass nor interfacial momentum (surface tension) is supplied.

Each term of the microscopic balance equations is averaged by using the procedure outlined in the previous section and presented in more detail in $[39,5]$. This leads to the $\alpha$-phase- $k$-material macroscopic conservation of mass

$$
\frac{\partial f^{k} \pi^{\alpha k} \rho^{\alpha k}}{\partial t}+\operatorname{div}\left(f^{k} \pi^{\alpha k} \rho^{\alpha k} \boldsymbol{v}^{\alpha k}\right)=\Lambda^{\alpha k}
$$


and macroscopic balance of momentum

$$
\begin{aligned}
& \frac{\partial f^{k} \pi^{\alpha k} \rho^{\alpha k} \boldsymbol{v}^{\alpha k}}{\partial t}+\operatorname{div}\left(f^{k} \pi^{\alpha k} \rho^{\alpha k} \boldsymbol{v}^{\alpha k} \otimes \boldsymbol{v}^{\alpha k}\right)= \\
& f^{k} \pi^{\alpha k} \rho^{\alpha k} \boldsymbol{b}^{\alpha k}+\operatorname{div}\left(f^{k} \pi^{\alpha k} \boldsymbol{\sigma}^{\alpha k}\right)+\Lambda^{\alpha k} \boldsymbol{v}_{\mathrm{I}}^{\mathrm{m}}+\boldsymbol{\Gamma}^{\alpha k},
\end{aligned}
$$

where

$$
\begin{gathered}
\Lambda^{\alpha k} \stackrel{\text { def }}{=}\left\langle\left(\rho\left(\boldsymbol{v}-\boldsymbol{v}_{\mathrm{I}}\right)\right)^{[\alpha k]} \cdot \boldsymbol{n}_{\mathrm{I}}^{\alpha k}\right\rangle, \\
\Lambda^{\alpha k} \boldsymbol{v}_{\mathrm{I}} \stackrel{\text { def }}{=}\left\langle\left(\rho \boldsymbol{v} \otimes\left(\boldsymbol{v}-\boldsymbol{v}_{\mathrm{I}}\right)\right)^{[\alpha k]} \cdot \boldsymbol{n}_{\mathrm{I}}^{\alpha k}\right\rangle, \\
\boldsymbol{\Gamma}^{\alpha k} \stackrel{\text { def }}{=}-\left\langle\boldsymbol{\sigma}^{[\alpha k]} \cdot \boldsymbol{n}_{\mathrm{I}}^{\alpha k}\right\rangle .
\end{gathered}
$$

The macroscopic field $\boldsymbol{v}^{\alpha k}$ is the spatial image of the material velocity, $\rho^{\alpha k}$ is the spatial mass density, $\boldsymbol{b}^{\alpha k}$ is a prescribed body force per unit mass, and $\boldsymbol{\sigma}^{\alpha k}=\left(\boldsymbol{\sigma}^{\alpha k}\right)^{\mathrm{T}}$ is the symmetric Cauchy stress. The latter is defined by the sum of the $\alpha$-phase- $k$-material intrinsic volume average of microscopic Cauchy stress and a residual stress, called Reynolds stress, due to mass flux relative to the averaging volume $[5$, sec. 11.2$] . q^{[\alpha k]}$ denotes the limit value of a field $q$ on the $\alpha k$-side of the interface, $\boldsymbol{v}_{\mathrm{I}}$ is the microscopic interface velocity, $\boldsymbol{v}_{\mathrm{I}}^{\mathrm{m}}$ is its averaged value compatible with (10) and (11), and $\boldsymbol{n}_{\mathrm{I}}^{\alpha k}$ is the field of normals on the interface pointing outward of the $\alpha$-phase- $k$-material.

The mass transfer term $\Lambda^{\alpha k}$ denotes the rate of mass supply per unit volume via the $\alpha$-phase- $k$-material interface. The momentum transfer term $\Lambda^{\alpha k} \boldsymbol{v}_{\mathrm{I}}^{\mathrm{m}}$ is due to transfer of inertial mass, whereas $\boldsymbol{\Gamma}^{\alpha k}$ includes surface drag forces per unit volume generated by the relative motion of the constituents. Total mass and momentum of the mixture is conserved, thus

$$
\begin{aligned}
\sum_{k} \sum_{\alpha} \Lambda^{\alpha k} & =0 \quad \text { and } \\
\sum_{k} \sum_{\alpha}\left(\Lambda^{\alpha k} \boldsymbol{v}_{\mathrm{I}}^{\mathrm{m}}+\boldsymbol{\Gamma}^{\alpha k}\right) & =\mathbf{0} .
\end{aligned}
$$


From this and the conditions (6), summation of (8) and (9) over all phases $\alpha \in\{1, \ldots, N\}$ and all materials $k \in\{1, \ldots, M\}$ finally yield the macroscopic conservation of mass and macroscopic balance of momentum of the mixture:

$$
\begin{gathered}
\frac{\partial\langle\rho\rangle}{\partial t}+\operatorname{div}\langle\rho \boldsymbol{v}\rangle=0 \quad \text { and } \\
\frac{\partial\langle\rho \boldsymbol{v}\rangle}{\partial t}+\operatorname{div}\langle\rho \boldsymbol{v} \otimes \boldsymbol{v}\rangle=\langle\rho \boldsymbol{b}\rangle+\operatorname{div}\langle\boldsymbol{\sigma}\rangle .
\end{gathered}
$$

Note that the averaged balance equations (8), (9), and (14) hold at each spatial point and at all interfaces, that is, a single spatial point is viewed as being simultaneously occupied by all materials and all phases of the mixture.

The present paper is particularly concerned with the flow situation illustrated in Fig. 2. Materials represent mixtures composed of a solid phase and a fluid phase such that $\alpha \in\{\mathrm{s}, \mathrm{f}\}$. Therefore, we simply define the fluid fraction or porosity of the $k$-material, $k \in\{\mathrm{S}, \mathrm{F}, \mathrm{G}\}$, through

$$
n^{k \stackrel{\text { def }}{=}} \pi^{\mathrm{f} k}
$$

The solid fraction within the $k$-material becomes $\pi^{\mathrm{s} k}=1-n^{k}$ by using $(5)_{2}$. In cases where the $k$-material consists of a solid without significant porosity $(k=\mathrm{S})$ one has $n^{\mathrm{S}}=0$. If on the other hand the $k$-material is a fluid $(k=\mathrm{F})$, then $n^{\mathrm{F}}=1$ applies. The mixture representing a single granular material is characterized by $0<n^{\mathrm{G}}<1$ and $f^{k} \equiv f^{\mathrm{G}}=1$.

\subsection{Lagrangian form of equations}

The equations of the previous section are in Eulerian form, referring to a fixed spatial reference volume instantaneously occupied by the material. However, the majority of methods for multi-material flow involve a Lagrangian step followed by a remap step to advance solution in time $[6,7]$; 
see also Sect. 5. Therefore, it proofs convenient to display the balance principles (8), (9), and (14) in the Lagrangian form.

First, let us define the material time derivative of a $\alpha$-phase- $k$-materialrelated quantity $q^{\alpha k}$ along the velocity $\boldsymbol{v}^{\alpha k}$ through

$$
\dot{q}^{\alpha k} \stackrel{\text { def }}{=} \frac{\partial q^{\alpha k}}{\partial t}+\boldsymbol{v}^{\alpha k} \cdot \nabla q^{\alpha k}
$$

The Lagrangian form of balance of momentum (9) is easily obtained under the assumption that mass is conserved. Clearly, substitution of (16) and (8) into (9), and using the product rule, yields

$$
\begin{aligned}
& f^{k} \pi^{\alpha k} \rho^{\alpha k} \dot{\boldsymbol{v}}^{\alpha k}= \\
& f^{k} \pi^{\alpha k} \rho^{\alpha k} \boldsymbol{b}^{\alpha k}+\operatorname{div}\left(f^{k} \pi^{\alpha k} \boldsymbol{\sigma}^{\alpha k}\right)+\Lambda^{\alpha k}\left(\boldsymbol{v}_{\mathrm{I}}^{\mathrm{m}}-\boldsymbol{v}^{\alpha k}\right)+\boldsymbol{\Gamma}^{\alpha k}
\end{aligned}
$$

By making use of the summation rules, balance of momentum of the mixture, $(14)_{2}$, in the Lagrangian form reads

$$
\langle\rho \dot{\boldsymbol{v}}\rangle=\langle\rho \boldsymbol{b}\rangle+\operatorname{div}\langle\boldsymbol{\sigma}\rangle
$$

in which we used the abbreviation

$$
\langle c \dot{q}\rangle \stackrel{\text { def }}{=} \sum_{k} \sum_{\alpha} f^{k} \pi^{\alpha k} c^{\alpha k} \dot{q}^{\alpha k}
$$

for arbitrary spatial fields $c, q$.

Concerning the $\alpha$-phase- $k$-material conservation of mass, substitution of (16) into (8) results in

$$
f^{k} \pi^{\alpha k} \dot{\rho}^{\alpha k}+\rho^{\alpha k}\left(\pi^{\alpha k} \dot{f}_{\alpha k}^{k}+f^{k} \dot{\pi}^{\alpha k}+f^{k} \pi^{\alpha k} \operatorname{div} \boldsymbol{v}^{\alpha k}\right)=\Lambda^{\alpha k},
$$

where $\dot{f}_{\alpha k}^{k}$ is shorthand for $\frac{\partial}{\partial t} f^{k}+\boldsymbol{v}^{\alpha k} \cdot \boldsymbol{\nabla} f^{k}$. By this, the Lagrangian form of $(14)_{1}$ representing conservation of mass of the mixture becomes

$$
\langle\dot{\rho}\rangle+\langle\rho\rangle \operatorname{div}\langle\boldsymbol{v}\rangle=0
$$


in which

$$
\begin{aligned}
& \langle\rho\rangle \operatorname{div}\langle\boldsymbol{v}\rangle \stackrel{\text { def }}{=} \\
& \sum_{k} \sum_{\alpha} \rho^{\alpha k}\left(\pi^{\alpha k} \dot{f}_{\alpha k}^{k}+f^{k} \dot{\pi}^{\alpha k}+f^{k} \pi^{\alpha k} \operatorname{div} \boldsymbol{v}^{\alpha k}\right) .
\end{aligned}
$$

\section{Phenomenological description of granular material}

\subsection{Some distinctive features of mechanical behavior}

One unique feature of geomechanical multi-material flow is the presence of geomaterial (e.g. soil, debris). The specific geomaterial of interest is a cohesionless granular material in which a single fluid fills the interstitial space. From a formal rheological viewpoint the material can be addressed as a dense grain-fluid mixture or "granular suspension" [43]. However, our terminology is more closely related to soil mechanics because we are particularly concerned with cohesionless soil (sand). The interstitial fluid can be gas, liquid, or a suspension of liquid and dispersed fines (slurry).

According to $[44,45,46,47]$, two limiting regimes of dry granular flow have to be considered. Under static or quasi-static loads the grains are in close contact and form a skeleton. The contact forces acting between the grains are dominated by the mean stress and dry friction (granular solid). Grain inertia effects are negligible, and the material response is rateindependent plastic. This is called the frictional or quasi-static regime, and it is the granular flow regime extensively studied in soil mechanics. In the quasistatic flow regime, the mechanical behavior of cohesionless granular material is very complex and has several distinctive features [47, 48, 49, 50, 51, 52]. Some of them are: 
- Internal friction: The shear strength increases with the mean effective stress, depending on the angle of internal friction.

- Dilatancy: Refers to the tendency to expand or contract in bulk volume because of grain rearrangement due to shear loading.

- Critical state: Under quasi-static monotonic shearing, an asymptotic state of stress and relative density is reached.

- State dependence: Dilatancy and plastic response depend on the states of stress and relative density.

- History dependence: The behavior of two specimen of the same granular material is different once they differ in material history.

At the other extreme characterized by high rates of shear deformation and smaller solid volume fractions, dry granular material behaves rate-dependent "viscous" (granular liquid). Grain inertia and instantaneous grain contacts through collision dominate [53], hence this flow regime is called the collisional or dynamic regime. In many practical flow situations frictional and collisional interactions are roughly of the same order, and the contributions of each to the bulk stress of the mixture cannot be clearly distinguished. Relatively little is known about this intermediate flow regime, called the frictionalcollisional regime, from both theoretical and experimental viewpoints [54, $55,56,57]$.

Interstitial fluid in granular materials introduces further complexity. Consolidation $[58,59,60]$ and liquefaction $[49,61]$ are two phenomena related to fluid-solid coupling which have been recognized for a long time. Besides 
this, indirect grain interactions generally occur through lubricated contacts $[43,62,55,57]$. Lubricated contact is characterized by repulsive viscous forces due to squeezing and shearing of the interstitial fluid. In general, all the mentioned flow regimes have to be considered in the analysis of geomechanical multi-material flow. However, the description of the mechanical behavior of a dense grain-fluid mixture for a wide range of flow conditions and material properties is still an open problem [57].

\subsection{Decompositions of stress}

In order to treat all materials that might be present in geomechanical multi-material flow (Figs. 1, 2) in a unified fashion, we recall from [41, 42] that the stress tensor of any material can be decomposed into a pressure stress and an extra stress according to

$$
\boldsymbol{\sigma}^{\alpha k}=-p^{\alpha k} \boldsymbol{I}+\boldsymbol{s}^{\alpha k}
$$

where $\boldsymbol{I}$ is the second-order unit tensor. We assume for simplicity that $p^{\alpha k}=$ $-\frac{1}{3} \operatorname{tr} \boldsymbol{\sigma}^{\alpha k}$ resp. $\boldsymbol{s}^{\alpha k}=\boldsymbol{\sigma}_{\mathrm{dev}}^{\alpha k}$ for all constituents, where $\operatorname{tr} \boldsymbol{t} \stackrel{\text { def }}{=} \boldsymbol{I}: \boldsymbol{t}$ returns the trace of a second-order tensor $\boldsymbol{t}$ and $\boldsymbol{t}_{\mathrm{dev}} \stackrel{\text { def }}{=} \boldsymbol{t}-\frac{1}{3}(\operatorname{tr} \boldsymbol{t}) \boldsymbol{I}$ is its deviator.

Based on (6) we assume that the bulk stress in granular material can be expressed as

$$
\boldsymbol{\sigma}^{\mathrm{G}}=\left(1-n^{\mathrm{G}}\right) \boldsymbol{\sigma}^{\mathrm{sG}}+n^{\mathrm{G}} \boldsymbol{\sigma}^{\mathrm{fG}}
$$

The stress tensors of the solid and fluid phases, on the other hand, can be represented as the linear sum of a rate-independent frictional contribution and a rate-dependent viscous contribution [44, 54, 46, 48, 63]:

$$
\boldsymbol{\sigma}^{\alpha \mathrm{G}} \stackrel{\text { def }}{=} \boldsymbol{\sigma}_{\mathrm{fr}}^{\alpha \mathrm{G}}+\boldsymbol{\sigma}_{\mathrm{vi}}^{\alpha \mathrm{G}}, \quad \text { with } \quad \alpha \in\{\mathrm{s}, \mathrm{f}\}
$$


so that $\boldsymbol{\sigma}^{\mathrm{G}}=\boldsymbol{\sigma}_{\mathrm{fr}}^{\mathrm{G}}+\boldsymbol{\sigma}_{\mathrm{vi}}^{\mathrm{G}}$ likewise.

Concerning the frictional part, we introduce Terzaghi's effective stress $\boldsymbol{\sigma}_{\mathrm{fr}}^{\mathrm{G}^{\prime}}$ in accordance with [64] as

$$
\begin{aligned}
& \frac{\boldsymbol{\sigma}_{\mathrm{fr}}^{\mathrm{G}^{\prime}}}{1-n^{\mathrm{G}}} \stackrel{\text { def }}{=}-\left(p^{\mathrm{sG}}-p^{\mathrm{fG}}\right) \boldsymbol{I}+\boldsymbol{s}_{\mathrm{fr}}^{\mathrm{sG}}, \text { so that } \\
& \frac{p^{\mathrm{G}^{\prime}}}{1-n^{\mathrm{G}}}=p^{\mathrm{sG}}-p^{\mathrm{fG}},
\end{aligned}
$$

where $p^{\mathrm{sG}}-p^{\mathrm{fG}}$ is called the excess pressure, $p^{\mathrm{G}^{\prime}} \stackrel{\text { def }}{=}-\frac{1}{3} \operatorname{tr} \boldsymbol{\sigma}_{\mathrm{fr}}^{\mathrm{G}^{\prime}}$ is the mean effective stress, and $\boldsymbol{s}_{\mathrm{fr}}^{\mathrm{sG}}=\left(\boldsymbol{\sigma}_{\mathrm{fr}}^{\mathrm{sG}}\right)_{\mathrm{dev}}$. Note that in a suspension without grain contacts each grain would be completely surrounded by water, resulting in $p^{\mathrm{sG}}=p^{\mathrm{fG}}$ and $p^{\mathrm{G}^{\prime}}=0$. Combination of (24) and (26) yields Terzaghi's principle of effective stress [50, 49, 65]

$$
\boldsymbol{\sigma}_{\mathrm{fr}}^{\mathrm{G}}=\boldsymbol{\sigma}_{\mathrm{fr}}^{\mathrm{G}^{\prime}}-p^{\mathrm{fG}} \boldsymbol{I}
$$

We adopt this particular form of the principle for conceptual reasons, while noting that various other forms have been postulated. The validity of each depends on the actual composition of material phases and on the pressure level, see [66] for a discussion.

In contrast to the frictional part, we assume that the effective stress for the collisional (dynamic) regime remains unaffected by fluid stresses. Therefore, by taking into account the principle (27) and (25), the representation (24) of the total Cauchy stress can be recast into

$$
\begin{aligned}
\boldsymbol{\sigma}^{\mathrm{G}} & =\boldsymbol{\sigma}_{\mathrm{fr}}^{\mathrm{G}^{\prime}}+\boldsymbol{\sigma}_{\mathrm{vi}}^{\mathrm{G}^{\prime}}+\boldsymbol{\sigma}_{\mathrm{fr}}^{\mathrm{fG}}+n^{\mathrm{G}} \boldsymbol{\sigma}_{\mathrm{vi}}^{\mathrm{fG}} \\
& =\boldsymbol{\sigma}^{\mathrm{G}^{\prime}}-p^{\mathrm{fG}} \boldsymbol{I}+n^{\mathrm{G}} \boldsymbol{s}^{\mathrm{fG}},
\end{aligned}
$$

with $\boldsymbol{\sigma}^{\mathrm{G}^{\prime}}=\boldsymbol{\sigma}_{\mathrm{fr}}^{\mathrm{G}^{\prime}}+\boldsymbol{\sigma}_{\mathrm{vi}}^{\mathrm{G}^{\prime}}$. We refer to (28) as the principle of effective stress 
for a general grain-fluid mixture. It has been used in [21], for example, to describe the flow of variably fluidized granular masses.

\subsection{Compressible constituents}

All constituents of that flows of interest are considered compressible. The finite bulk modulus, $K$, of a single-phase, isotropic material is the inverse of the compressibility and defined by

$$
-\left.\frac{1}{V} \frac{\partial V}{\partial p}\right|_{M}=\frac{1}{\rho} \frac{\mathrm{d} \rho}{\mathrm{d} p} \stackrel{\text { def }}{=} \frac{1}{K} \quad \text { resp. } \quad \dot{p} \stackrel{\text { def }}{=} \frac{K}{\rho} \dot{\rho},
$$

where $V$ and $M \stackrel{\text { def }}{=} \rho V$ are the total volume and mass, respectively, of a sufficiently large domain, and $\left.\right|_{M}$ means that mass is kept constant along with differentiation.

In the above we are implicitly assuming that $V=V(p, M)$, or equivalently, that total pressure is a function of the density only. However, in a mixture the pressure of each compressible constituent is generally a function of the mass density and volume fraction of that constituent. In particular, the pressure of the solid phase in granular material does not only depend on the mass density but also on the porosity $[67,68]$. If the granular material occupies only a fractional volume in a mixture of bulk materials, the pressure of the solid phase of the granular material should moreover depend on $f^{\mathrm{G}}$.

Instead of rigorously deriving relationships between the various compressibilities, pressures, and volume fractions associated with geomechanical multi-material flow, we adopt a simplified procedure presented in [69] and repeated in [68]. The basic assumption is $V^{\mathrm{G}}=V^{\mathrm{G}}\left(p^{\mathrm{fG}}, p^{\mathrm{G}}-p^{\mathrm{fG}}\right)$ while keeping the total mass of the solid phase $M^{\text {sG }}$ fixed. That is to say, the total 
volume $V^{\mathrm{G}}=V^{\mathrm{sG}}+V^{\mathrm{fG}}$ of a granular material is a function of two independent pressures: the pressure of the fluid phase, $p^{\mathrm{fG}}$, and the difference between the total pressure and the fluid pressure, $p^{\mathrm{G}}-p^{\mathrm{fG}}$, with no solid leaving the system. From this we get

$$
\begin{aligned}
& \left.\frac{\mathrm{d} V^{\mathrm{G}}}{V^{\mathrm{G}}}\right|_{M^{\mathrm{sG}}}=\left.\frac{1}{V^{\mathrm{G}}} \frac{\partial V^{\mathrm{G}}}{\partial\left(p^{\mathrm{G}}-p^{\mathrm{fG}}\right)}\right|_{p^{\mathrm{fG},}, M^{\mathrm{sG}}} \mathrm{d}\left(p^{\mathrm{G}}-p^{\mathrm{fG}}\right) \\
& +\left.\frac{1}{V^{\mathrm{G}}} \frac{\partial V^{\mathrm{G}}}{\partial p^{\mathrm{fG}}}\right|_{p^{\mathrm{G}}-p^{\mathrm{fG}, M^{\mathrm{sG}}}} \mathrm{d} p^{\mathrm{fG}} \\
& =\left.\frac{1}{V^{\mathrm{G}}} \frac{\partial V^{\mathrm{G}}}{\partial p^{\mathrm{G}}}\right|_{p^{\mathrm{fG}, M^{\mathrm{sG}}}} \mathrm{d}\left(p^{\mathrm{G}}-p^{\mathrm{fG}}\right) \\
& +\left.\frac{1}{V^{\mathrm{G}}} \frac{\partial V^{\mathrm{G}}}{\partial p^{\mathrm{fG}}}\right|_{p^{\mathrm{G}}-p^{\mathrm{fG}, M^{\mathrm{sG}}}} \mathrm{d} p^{\mathrm{fG}} \\
& \stackrel{\text { def }}{=}-\frac{1}{K_{\mathrm{dr}}^{\mathrm{G}}} \mathrm{d}\left(p^{\mathrm{G}}-p^{\mathrm{fG}}\right)-\frac{1}{K_{\mathrm{uj}}^{\mathrm{G}}} \mathrm{d} p^{\mathrm{fG}},
\end{aligned}
$$

in which $p^{\mathrm{G}} \stackrel{\text { def }}{=}-\frac{1}{3} \operatorname{tr} \boldsymbol{\sigma}_{\mathrm{fr}}^{\mathrm{G}}$, and $K_{\mathrm{dr}}^{\mathrm{G}}$ and $K_{\mathrm{uj}}^{\mathrm{G}}$ are referred to as the drained bulk modulus and unjacketed bulk modulus of the granular material, respectively [67]. $K_{\mathrm{uj}}^{\mathrm{G}}$ is measured during an unjacketed test and is approximately equal to $K^{\mathrm{sG}}=\rho^{\mathrm{sG}} \partial p^{\mathrm{sG}} / \partial \rho^{\mathrm{sG}}$, the material bulk modulus of the solid phase, under the assumption of constant solid phase volume fraction. $K_{\mathrm{dr}}^{\mathrm{G}}$ is the bulk modulus of the drained granular material as measured in a jacketed test.

The total differential in (30) can be replaced with the material time derivative along the velocity $\boldsymbol{v}^{\mathrm{sG}}$. Then, by noting that $M^{\mathrm{sG}}=\rho^{\mathrm{sG}} V^{\mathrm{sG}}=$ $\left(1-n^{\mathrm{G}}\right) \rho^{\mathrm{sG}} V^{\mathrm{G}} \stackrel{\text { def }}{=} \tilde{\rho}^{\mathrm{sG}} V^{\mathrm{G}}$, where $\tilde{\rho}^{\mathrm{sG}}$ is the bulk mass density of the dry granular material, conservation of mass (8) resp. (20) yields the identity

$$
\left.\frac{\dot{V}^{\mathrm{G}}}{V^{\mathrm{G}}}\right|_{M^{\mathrm{sG}}}=-\frac{\dot{\tilde{\rho}}^{\mathrm{SG}}}{\tilde{\rho}^{\mathrm{SG}}}=\operatorname{div} \boldsymbol{v}^{\mathrm{sG}}+\frac{\dot{f}_{\mathrm{SG}}^{\mathrm{G}}}{f^{\mathrm{G}}} \stackrel{\operatorname{def}}{=} \operatorname{div} \boldsymbol{v}_{*}^{\mathrm{sG}}
$$


By this, (30) after rearrangement takes the equivalent form

$$
\dot{p}^{\mathrm{G}}=\dot{p}^{\mathrm{G}^{\prime \prime}}+\zeta^{\mathrm{G}} \dot{p}^{\mathrm{fG}}, \quad \text { where } \zeta^{\mathrm{G}} \stackrel{\text { def }}{=} 1-\frac{K_{\mathrm{dr}}^{\mathrm{G}}}{K_{\mathrm{uj}}^{\mathrm{G}}}
$$

is a coefficient introduced by Biot and Willis [70], and $\dot{p}^{\mathrm{G}^{\prime \prime}} \stackrel{\text { def }}{=}-K_{\mathrm{dr}}^{\mathrm{G}} \operatorname{div} \boldsymbol{v}_{*}^{\mathrm{sG}}$. We use the approximation $K_{\mathrm{uj}}^{\mathrm{G}} \approx K^{\mathrm{sG}}$ in what follows.

From (32), (24), and (26) one obtains

$$
\dot{p}^{\mathrm{G}^{\prime}}=\dot{p}^{\mathrm{G}^{\prime \prime}}-\frac{K_{\mathrm{dr}}^{\mathrm{G}}}{K^{\mathrm{sG}}} \dot{p}^{\mathrm{fG}}=-K_{\mathrm{dr}}^{\mathrm{G}}\left(\operatorname{div} \boldsymbol{v}_{*}^{\mathrm{sG}}+\frac{\dot{p}^{\mathrm{fG}}}{K^{\mathrm{sG}}}\right) .
$$

Moreover, a derivation similar to (30), but starting from the relative volume change $\mathrm{d} V^{\mathrm{sG}} /\left.V^{\mathrm{sG}}\right|_{1-n^{\mathrm{G}}}$ and using the relationships above, yields an equation for the solid phase pressure in granular material:

$$
\dot{p}^{\mathrm{SG}}=-\frac{K_{\mathrm{dr}}^{\mathrm{G}}}{1-n^{\mathrm{G}}} \operatorname{div} \boldsymbol{v}_{*}^{\mathrm{sG}}+\frac{\zeta^{\mathrm{G}}-n^{\mathrm{G}}}{1-n^{\mathrm{G}}} \dot{p}^{\mathrm{fG}} .
$$

Under the assumption of locally undrained conditions, for which

$$
\boldsymbol{v}^{\mathrm{fG}}=\boldsymbol{v}^{\mathrm{sG}}=\boldsymbol{v}^{\mathrm{G}}, \text { but } p^{\mathrm{fG}} \neq p^{\mathrm{sG}} \quad \text { in general, }
$$

conservation of mass (20) for the fluid phase is equivalent to

$$
\dot{p}^{\mathrm{fG}}=-\zeta^{\mathrm{G}} Q^{\mathrm{G}} \operatorname{div} \boldsymbol{v}_{*}^{\mathrm{sG}}
$$

with

$$
Q^{\mathrm{G}} \stackrel{\text { def }}{=}\left(\frac{\zeta^{\mathrm{G}}-n^{\mathrm{G}}}{K^{\mathrm{sG}}}+\frac{n^{\mathrm{G}}}{K^{\mathrm{fG}}}\right)^{-1}
$$

Substitution of (36) into (32) finally yields

$$
\dot{p}^{\mathrm{G}}=-K^{\mathrm{G}} \operatorname{div} \boldsymbol{v}_{*}^{\mathrm{sG}},
$$


in which

$$
K^{\mathrm{G}}=K_{\mathrm{dr}}^{\mathrm{G}}\left(1+\frac{\left(\zeta^{\mathrm{G}}\right)^{2}}{\zeta^{\mathrm{G}} \frac{K_{\mathrm{dr}}^{\mathrm{G}}}{K^{\mathrm{sG}}}+n^{\mathrm{G}}\left(\frac{K_{\mathrm{dr}}^{\mathrm{G}}}{K^{\mathrm{fG}}}-\frac{K_{\mathrm{dr}}^{\mathrm{G}}}{K^{\mathrm{sG}}}\right)}\right) .
$$

Compressibility relates a change in pressure to a change in volume. Thus the pressure of one material in a mixture is affected by the presence of the other materials through the change in fractional volume. The derivations of this section particularly show that the rates of different pressures in granular

material share the same functional dependence on $\dot{f}_{\mathrm{sG}}^{\mathrm{G}}=\operatorname{div}\left(\boldsymbol{v}_{*}^{\mathrm{sG}}-\boldsymbol{v}^{\mathrm{sG}}\right) f^{\mathrm{G}}$. Readers may recover the classical relations of a single porous medium with compressible constituents $[71,72,49]$ from those presented here for the case where $f^{\mathrm{G}} \equiv 1$, so that $\dot{f}_{\mathrm{sG}}^{\mathrm{G}}=0$.

\section{Homogeneous equilibrium model}

The set of balance equations derived in Sect. 2.2 needs to be closed in order to describe the flow of interest illustrated in Fig. 2. In general, the following closure relations have to be specified [38, 73]:

1. Transfer relations expressing the physics at the material interfaces.

2. Constitutive relations characterizing the physical behavior of each material.

3. Topological relations accounting for the evolution of variables characterizing the interfacial structure.

The crucial relations are those governing the evolution of the volume fractions $f^{k}$. In the present paper, we achieve closure by a priori assuming 
homogeneous equilibrium $[4,16,17]$. In particular,

$$
p^{k}=\langle p\rangle \quad \text { and } \quad \boldsymbol{v}^{k}=\langle\boldsymbol{v}\rangle \quad \text { for all } k \in\{\mathrm{S}, \mathrm{F}, \mathrm{G}\}
$$

\subsection{Interfacial transfer closure relations}

The presence of interfacial transfer is a basic property of multi-phase and multi-fluid flows. Therefore, a large body of literature concerned with suitable transfer relations for particular types of flows is available $[2,3,5,74]$. Here we assume for simplicity that neither mass nor momentum is exchanged no matter between which constituents, so that

$$
\Lambda^{\alpha k}=0 \quad \text { and } \quad \boldsymbol{\Gamma}^{\alpha k}=\mathbf{0}
$$

for all $\alpha \in\{\mathrm{s}, \mathrm{f}\}$ and $k \in\{\mathrm{S}, \mathrm{F}, \mathrm{G}\}$.

\subsection{Constitutive closure relations}

It is beyond the scope of the paper to provide and discuss in detail constitutive equations for the bulk solid, bulk fluid, and fluid-saturated granular material. We only give hints on what classes of equations might be suitable and assume that the reader is somewhat familiar with the principles of constitutive theory. The latter are treated, for example, in [41, 42, 5, 75].

The objective is to determine for each material $k \in\{\mathrm{S}, \mathrm{F}, \mathrm{G}\}$ the total stress given by $\boldsymbol{\sigma}^{k}=\sum_{\alpha} \pi^{\alpha k} \boldsymbol{\sigma}^{\alpha k}=\left(1-n^{k}\right) \boldsymbol{\sigma}^{\mathrm{sk}}+n^{k} \boldsymbol{\sigma}^{\mathrm{f} k}$, with $\boldsymbol{\sigma}_{\mathrm{dev}}^{\alpha k}=\boldsymbol{s}^{\alpha k}$ and the fluid fraction being either $n^{\mathrm{S}}=0$ in case of bulk solid, $n^{\mathrm{F}}=1$ in case of bulk fluid, or $0<n^{\mathrm{G}}<1$ in case of fluid-saturated granular material. The averaged stress tensor of the mixture then can be calculated from $\langle\boldsymbol{\sigma}\rangle=\sum_{k} f^{k} \boldsymbol{\sigma}^{k}=f^{\mathrm{S}} \boldsymbol{\sigma}^{\mathrm{S}}+f^{\mathrm{F}} \boldsymbol{\sigma}^{\mathrm{F}}+f^{\mathrm{G}} \boldsymbol{\sigma}^{\mathrm{G}}$. 


\subsubsection{Bulk fluid and bulk solid}

For bulk materials, various constitutive approaches are available. In our approach, bulk fluid is represented as a Newtonian fluid. Bulk solid is either hypoelastic or hypoelasto-plastic, described by a rate constitutive equation taking the general form $[42,76,77,49]$

$$
\stackrel{\nabla}{\mathrm{\sigma}} \stackrel{\text { def }}{=} \boldsymbol{c}^{\mathrm{S}}\left(\boldsymbol{h}^{\mathrm{S}}\right):\langle\boldsymbol{d}\rangle
$$

where $\boldsymbol{c}^{\mathrm{S}}$ is a fourth-order material tangent tensor, $\boldsymbol{h}^{\mathrm{S}} \stackrel{\text { def }}{=}\left\{h_{1}^{\mathrm{S}}, \ldots, h_{m}^{\mathrm{S}}\right\}$ is a (probably empty) set of material state variables, $\stackrel{\nabla}{\stackrel{\text { def }}{=}} \boldsymbol{\sigma}+\boldsymbol{\sigma} \cdot \boldsymbol{\omega}-\boldsymbol{\omega} \cdot \boldsymbol{\sigma}$ denotes the Zaremba-Jaumann rate of the considered second order tensor, $\boldsymbol{\omega} \stackrel{\text { def }}{=} \frac{1}{2}\left(\boldsymbol{\nabla} \boldsymbol{v}-(\boldsymbol{\nabla} \boldsymbol{v})^{\mathrm{T}}\right)$ is the vorticity tensor, and $\boldsymbol{d} \stackrel{\text { def }}{=} \frac{1}{2}\left(\boldsymbol{\nabla} \boldsymbol{v}+(\boldsymbol{\nabla} \boldsymbol{v})^{\mathrm{T}}\right)$ is the spatial rate of deformation tensor.

The simplest model represented by (42) is hypoelasticty of grade zero $[78,41]$

$$
\stackrel{\nabla}{\mathrm{\sigma}} \stackrel{\text { def }}{=} \boldsymbol{c}_{\mathrm{e}}^{\mathrm{S}}:\langle\boldsymbol{d}\rangle=K \operatorname{tr}\langle\boldsymbol{d}\rangle \boldsymbol{I}+2 G\langle\boldsymbol{d}\rangle_{\mathrm{dev}}
$$

in which $K$ and $G$ are the constant bulk modulus and shear modulus, respectively, and $\boldsymbol{c}_{\mathrm{e}}^{\mathrm{S}}$ is the constant isotropic elasticity tensor. Despite its lack to represent properly elastic behavior [79], (43) remains widely used in computational solid mechanics, especially with reference to inelasticity [75, 77, 49].

Rate-independent elasto-plastic behavior has been traditionally described by proposing an additive decomposition of spatial rate of deformation into elastic and plastic parts: $\boldsymbol{d}^{\mathrm{S}} \stackrel{\text { def }}{=} \boldsymbol{d}_{\mathrm{e}}^{\mathrm{S}}+\boldsymbol{d}_{\mathrm{p}}^{\mathrm{S}}$. A hypoelastic constitutive relation then characterizes the elastic response, e.g. $\stackrel{\nabla}{\boldsymbol{\sigma}} \stackrel{\text { def }}{=} \boldsymbol{c}_{\mathrm{e}}^{\mathrm{S}}:\left(\langle\boldsymbol{d}\rangle-\boldsymbol{d}_{\mathrm{p}}^{\mathrm{S}}\right)$ by using (43). The yield condition $y\left(\boldsymbol{\sigma}^{\mathrm{S}}, \ldots\right) \leq 0$ defines the set of admissible states in stress space. An admissible state satisfying $y<0$ is an elastic state, whereas 
$y=0$ is an elasto-plastic state lying on the yield surface. As a simple example we consider the von Mises yield condition

$$
y\left(\boldsymbol{\sigma}^{\mathrm{S}}, \sigma^{\mathrm{y}}\right) \stackrel{\text { def }}{=} \sqrt{3 J_{2}}-\sigma^{\mathrm{y}},
$$

where $\sqrt{3 J_{2}\left(\boldsymbol{\sigma}^{\mathrm{S}}\right)} \stackrel{\text { def }}{=} \sqrt{\frac{3}{2}}\left\|\boldsymbol{\sigma}_{\mathrm{dev}}^{\mathrm{S}}\right\|$ is the von Mises stress, $\|\cdot\|$ is the Frobenius norm of a second-order tensor, and $\sigma^{\mathrm{y}}$ is the yield stress in pure tension. For $\sigma^{\mathrm{y}}=$ const the behavior is ideal plastic. Condition (44) applies to metals or plastic soil with zero internal friction and describes a cylinder around the trisectrix $\sigma_{1}=\sigma_{2}=\sigma_{3}$, with $\sigma_{1}, \sigma_{2}, \sigma_{3}$ being the eigenvalues of $\boldsymbol{\sigma}^{\mathrm{S}}$ (principal stresses).

The evolution of the plastic strain rate, $\boldsymbol{d}_{\mathrm{p}}^{\mathrm{S}}$, is prescribed by a flow rule,

$$
\boldsymbol{d}_{\mathrm{p}}^{\mathrm{S}} \stackrel{\text { def }}{=} \lambda \frac{\partial g}{\partial \boldsymbol{\sigma}^{\mathrm{S}}}
$$

in which $g\left(\boldsymbol{\sigma}^{\mathrm{S}}, \ldots\right)$ is called the plastic potential and $\lambda \geq 0$ is the consistency parameter. An associated flow rule is obtained for $g=y$. The hypoelasto-plastic constitutive relation is completed by determining $\lambda$ from the consistency condition

$$
\lambda \dot{y}\left(\boldsymbol{\sigma}^{\mathrm{S}}, \ldots\right)=0 .
$$

\subsubsection{Fluid-saturated granular material}

Concerning fluid-saturated granular material, constitutive equations must be specified for the fluid phase stress and for the frictional and viscous parts of the effective stress; cf. Sect. 3. For simplicity, the fluid phase (pore resp. interstitial fluid) is modeled as a Newtonian fluid with deviatoric viscous stress. In order to account for the fact that the fluid phase is trapped in the granular 
material we define the dynamic shear viscosity through $\mu^{\mathrm{fG}} \stackrel{\text { def }}{=}\left(n^{\mathrm{G}}\right)^{2} \mu_{0}^{\mathrm{fG}}$ in accordance with [80], where $\mu_{0}^{\mathrm{fG}}$ is the shear viscosity of the fluid for $n^{\mathrm{G}}=1$. The rate of the fluid phase pressure is determined from (36). The bulk modulus, $K^{\mathrm{fG}}$, and hence the pressure change are usually negligible if the fluid phase is a gas. If the fluid phase is a liquid, then $K^{\mathrm{fG}}$ generally depends on pressure, temperature, gas content, and content of dispersed fines.

Different approaches can be employed to model the mechanical behavior of bulk granular materials. Phenomenological constitutive equations predicting the effective stress, $\boldsymbol{\sigma}^{\mathrm{G}^{\prime}}=\boldsymbol{\sigma}_{\mathrm{fr}}^{\mathrm{G}^{\prime}}+\boldsymbol{\sigma}_{\mathrm{vi}}^{\mathrm{G}^{\prime}}$, are eminently suited to explore fundamental behavior of complex phenomena in geomechanics $[48,49,57,81$, 52]. However, an equation accounting for all features of granular material behavior in the full frictional-collisional regime is still out of reach.

A large number of constitutive relations has been proposed to model the quasi-static frictional regime for applications in soil mechanics. States of failure can be adequately modeled by elasto-plastic models employing the classical Mohr-Coulomb and Drucker-Prager yield conditions [49, 65, 82]. The yield condition of Mohr-Coulomb,

$$
\tau_{\mathrm{f}} \stackrel{\text { def }}{=} \sigma^{\prime} \tan \phi
$$

relates the shear stress at failure, $\tau_{\mathrm{f}}$, to the effective normal stress component $\sigma^{\prime}$ and the angle of internal friction of the bulk material, $\phi$. The latter is not directly related to the Coulomb frictional behavior at the grain scale, but is significantly influenced by grain interlocking effects resulting from the arrangement and angularity of the grains [65, 57]. The Drucker-Prager yield condition, on the other hand, describes a cone around the line of equal principal stresses, with the apex lying at $\sigma_{1}=\sigma_{2}=\sigma_{3}=0$ for cohesionless 
soil or debris material:

$$
y\left(\boldsymbol{\sigma}_{\mathrm{fr}}^{\mathrm{G}^{\prime}}\right) \stackrel{\text { def }}{=} \sqrt{3 J_{2}}+\beta I_{1} / 3, \quad \text { with } I_{1}\left(\boldsymbol{\sigma}_{\mathrm{fr}}^{\mathrm{G}^{\prime}}\right) \stackrel{\text { def }}{=} \operatorname{tr} \boldsymbol{\sigma}_{\mathrm{fr}}^{\mathrm{G}^{\prime}} .
$$

The factor $\beta$ is a material constant accounting for the mean effective stress sensitivity. It is related to the Mohr-Coulomb friction angle through $\beta=$ $2 \sin \phi /(3-\sin \phi)$.

Beyond states of failure in the frictional flow regime, comprehensive stressstrain relations for granular bulk material have to account for dilatancy, state dependence, and critical state behavior (cf. Sect. 3.1). In this context not many models are available, and most of them fall into the categories of hypoelasto-plastic $[83,84,85,86]$ or hypoplastic $[87,88,89,90,91]$ rate constitutive equations. They determine an objective rate of the effective stress as a function of the rate of deformation, the effective stress, the porosity $n^{\mathrm{G}}$ or void ratio $e^{\mathrm{G}} \stackrel{\text { def }}{=} n^{\mathrm{G}} /\left(1-n^{\mathrm{G}}\right)$, and a (probably empty) set of additional state variables $\boldsymbol{h}^{\mathrm{G}}$.

We assume for simplicity that $\boldsymbol{\nabla} n^{\mathrm{G}} \equiv \mathbf{0}$ and consider the generic relation

$$
\stackrel{\nabla}{\mathrm{fr}}_{\mathrm{G}^{\prime}} \stackrel{\text { def }}{=} \boldsymbol{c}_{\mathrm{fr}}^{\mathrm{G}^{\prime}}\left(\boldsymbol{\sigma}_{\mathrm{fr}}^{\mathrm{G}^{\prime}}, n^{\mathrm{G}}, \boldsymbol{h}^{\mathrm{G}}\right):\left(\langle\boldsymbol{d}\rangle+\frac{1}{3} \dot{p}^{\mathrm{fG}} / K^{\mathrm{sG}} \boldsymbol{I}\right) .
$$

Note that rates of volumetric strain resulting from pore pressure changes, represented by $-\dot{p}^{\mathrm{fG}} / K^{\mathrm{sG}}$, are not taken into account because they do not alter the arrangement of grains. This is in accordance with (33). To give a particular example for (49), we chose a hypoplastic model frequently applied in the German and international soil mechanics and geotechnical engineering community. The full mathematical expressions of the constitutive functions are attached in the Appendix. 
The majority of constitutive relations for the dynamic contribution of the effective bulk stress in granular material, $\boldsymbol{\sigma}_{\mathrm{vi}}^{\mathrm{G}^{\prime}}$, are restricted to particular flow conditions or to narrow ranges of material properties. In the present research, we adopt a simple model formulation suggested by Passman et al. [80] and further investigated in $[92,63]$. Its representation in rate form has been used in $[93,94]$ :

$$
{\stackrel{\nabla}{\mathrm{v}^{\mathrm{v}}}}_{\mathrm{G}}^{\mathrm{G}^{\prime}} \stackrel{\text { def }}{=} 2 \mu^{\mathrm{G}^{\prime}}\langle\stackrel{\nabla}{\boldsymbol{d}}\rangle_{\mathrm{dev}}
$$

Volume viscosity is currently neglected. The dynamic shear viscosity $\mu^{\mathrm{G}^{\prime}}$ is generally a function of the porosity (solid concentration) and shear rate. Relations have been proposed for different flow situations resp. flow geometries, e.g. $[53,95,80,54,48,94,81]$.

\subsection{Topological closure relations}

The application of volume averaging entails a loss of information as it smoothes out details of the flow structure [38, 73]. Topological closure relations are required to restore the lost information. Concerning the present situation, such relations have to describe the evolution of the porosity $n^{\mathrm{G}}$ of granular material and of the material volume fractions $f^{k}$, whereas $\dot{n}^{\mathrm{S}}=$ $\dot{n}^{\mathrm{F}} \equiv 0$.

A proper closure relation for volume fraction has to account for the physics of the problem and particularly has to specify how the volumetric distribution of the materials change. Although several relations have been formulated for the evolution of volume fractions in gas-liquid flows $[73,5,3]$ or the compaction of porous materials [96, 97, 98, 88], these do not automatically carry over to the present situation. 
A simple way to obtain topological closure for multi-material flow is to assume constant volume fractions. However, such an assumption is inadequate for the flows of interest since material compressibilities may differ by several orders of magnitude, e.g. if the bulk fluid represents air. In following $[16,17]$, a more realistic closure model can be derived which uses the assumptions (40). To this end, conservation of mass (8) resp. (20) for single-phase $k$-material without mass transfer is rearranged to get

$$
\dot{f}^{k}+f^{k} \operatorname{div}\langle\boldsymbol{v}\rangle=-f^{k} \frac{\dot{\rho}^{k}}{\rho^{k}} .
$$

The assumption (40) 1 and the general definition (19) yield

$$
\left\langle\frac{\dot{p}}{K}\right\rangle=\sum_{k} \frac{f^{k} \dot{p}^{k}}{K^{k}}=\frac{\langle\dot{p}\rangle}{\langle K\rangle}, \quad \text { with } \frac{1}{\langle K\rangle}=\sum_{k} \frac{f^{k}}{K^{k}} .
$$

Moreover, the compression model (29) gives

$$
\frac{K^{k}}{\rho^{k}} \dot{\rho}^{k}=\dot{p}^{k}=\langle\dot{p}\rangle=\left\langle\frac{K}{\rho} \dot{\rho}\right\rangle=\langle K\rangle\left\langle\frac{\dot{\rho}}{\rho}\right\rangle .
$$

By this, (51) in conjunction with the Lagrangian form of conservation of mass of the mixture, (21), result in the self-consistent balance equation

$$
\dot{f}^{k}+f^{k} \operatorname{div}\langle\boldsymbol{v}\rangle=-f^{k} \frac{\langle K\rangle}{K^{k}}\left\langle\frac{\dot{\rho}}{\rho}\right\rangle=f^{k} \frac{\langle K\rangle}{K^{k}} \operatorname{div}\langle\boldsymbol{v}\rangle .
$$

Hence, the topological closure relation for the $k$-material volume fraction is

$$
\dot{f}^{k}=f^{k}\left(\frac{\langle K\rangle}{K^{k}}-1\right) \operatorname{div}\langle\boldsymbol{v}\rangle .
$$

An evolution equation for the porosity of granular material can be derived from the mass conservation equation (20) by employing the assumptions of homogeneous velocity and zero mass transfer:

$$
\dot{n}^{\mathrm{G}}=\left(1-n^{\mathrm{G}}\right)\left(\frac{\dot{p}^{\mathrm{sG}}}{K^{\mathrm{sG}}}+\frac{\dot{f}^{\mathrm{G}}}{f^{\mathrm{G}}}+\operatorname{div}\langle\boldsymbol{v}\rangle\right) .
$$


The sum of the last two terms in parentheses represent the effective volumetric strain rate of the bulk granular material at constant mass and intrinsic mass density (no change of solid phase volume $V^{\mathrm{sG}}$ ). Thus we define $\operatorname{div}\left\langle\boldsymbol{v}_{\mathrm{G}}\right\rangle \stackrel{\text { def }}{=} \operatorname{div}\langle\boldsymbol{v}\rangle+\dot{f}^{\mathrm{G}} / f^{\mathrm{G}}$ in accordance with (31), or, more generally,

$$
\left\langle\boldsymbol{d}_{k}\right\rangle \stackrel{\text { def }}{=}\langle\boldsymbol{d}\rangle+\frac{\dot{f}^{k}}{3 f^{k}} \boldsymbol{I}, \quad \text { for all } k \in\{\mathrm{S}, \mathrm{F}, \mathrm{G}\},
$$

by assuming an isotropic rate of change in material volume fraction and using the identity $\operatorname{div} \boldsymbol{v}=\operatorname{tr} \boldsymbol{d}$. Obviously, $\left\langle\boldsymbol{d}_{k}\right\rangle$ rather than $\langle\boldsymbol{d}\rangle$ should be used in the constitutive closure relations addressed in Sect. 4.2.

\subsection{Summary of the model}

In combining the macroscopic balance principles derived in Sect. 2 with the particularizations made for saturated granular material in Sect. 3, and by using the assumptions (40), (35), and the closure relations specified in the previous sections, the following homogeneous equilibrium mixture model for geomechanical multi-material flow is obtained:

$$
\begin{aligned}
\operatorname{div}\langle\boldsymbol{s}-p \boldsymbol{I}\rangle+\langle\rho \boldsymbol{b}\rangle-\langle\rho\rangle\langle\dot{\boldsymbol{v}}\rangle & =\mathbf{0} \\
\langle\dot{p}\rangle /\langle K\rangle+\operatorname{div}\langle\boldsymbol{v}\rangle & =0,
\end{aligned}
$$

where

$$
\begin{aligned}
\langle\boldsymbol{s}\rangle & =\sum_{k} f^{k} \boldsymbol{s}^{k}=f^{\mathrm{S}} \boldsymbol{s}^{\mathrm{S}}+f^{\mathrm{F}} \boldsymbol{s}^{\mathrm{F}}+f^{\mathrm{G}}\left(\boldsymbol{s}^{\mathrm{G}^{\prime}}+n^{\mathrm{G}} \boldsymbol{s}^{\mathrm{fG}}\right), \\
\langle p\rangle & =\sum_{k} f^{k} p^{k}=f^{\mathrm{S}} p^{\mathrm{S}}+f^{\mathrm{F}} p^{\mathrm{F}}+f^{\mathrm{G}}\left(p^{\mathrm{G}^{\prime}}+p^{\mathrm{fG}}\right) \\
\langle\rho\rangle & =\sum_{k} f^{k} \rho^{k} \\
& =f^{\mathrm{S}} \rho^{\mathrm{S}}+f^{\mathrm{F}} \rho^{\mathrm{F}}+f^{\mathrm{G}}\left(\left(1-n^{\mathrm{G}}\right) \rho^{\mathrm{sG}}+n^{\mathrm{G}} \rho^{\mathrm{fG}}\right)
\end{aligned}
$$




$$
\begin{gathered}
\frac{1}{\langle K\rangle}=\sum_{k} \frac{f^{k}}{K^{k}}=\frac{f^{\mathrm{S}}}{K^{\mathrm{S}}}+\frac{f^{\mathrm{F}}}{K^{\mathrm{F}}}+\frac{f^{\mathrm{G}}}{K^{\mathrm{G}}}, \\
K^{\mathrm{G}}=K_{\mathrm{dr}}^{\mathrm{G}}\left(\begin{array}{c}
\zeta^{\mathrm{G}} \frac{K_{\mathrm{dr}}^{\mathrm{G}}}{K^{\mathrm{sG}}}+n^{\mathrm{G}}\left(\frac{K_{\mathrm{dr}}^{\mathrm{G}}}{K^{\mathrm{fG}}}-\frac{K_{\mathrm{dr}}^{\mathrm{G}}}{K^{\mathrm{sG}}}\right)
\end{array}\right), \\
\zeta^{\mathrm{G}}=1-\frac{K_{\mathrm{dr}}^{\mathrm{G}}}{K^{\mathrm{sG}}}, \quad \text { and } \quad k \in\{\mathrm{S}, \mathrm{F}, \mathrm{G}\} .
\end{gathered}
$$

The model is closed by the general constitutive equations for the

a) bulk solid:

$$
\stackrel{\nabla}{\boldsymbol{\sigma}}^{\mathrm{S}}=\boldsymbol{c}^{\mathrm{S}}\left(\boldsymbol{\sigma}^{\mathrm{S}}, \boldsymbol{h}^{\mathrm{S}}\right):\left\langle\boldsymbol{d}_{\mathrm{S}}\right\rangle, \quad K^{\mathrm{S}}=\frac{1}{3} \boldsymbol{I}: \boldsymbol{c}^{\mathrm{S}}: \boldsymbol{I},
$$

b) bulk fluid:

$$
\boldsymbol{\sigma}^{\mathrm{F}}=-p^{\mathrm{F}} \boldsymbol{I}+2 \mu^{\mathrm{F}}\left\langle\boldsymbol{d}_{\mathrm{F}}\right\rangle_{\operatorname{dev}}, \quad \dot{p}^{\mathrm{F}}=-K^{\mathrm{F}} \operatorname{div}\left\langle\boldsymbol{v}_{\mathrm{F}}\right\rangle,
$$

c) granular material:

$$
\begin{aligned}
& \stackrel{\nabla}{\boldsymbol{\sigma}}^{\mathrm{G}^{\prime}}=\stackrel{\nabla}{\mathrm{f}}_{\mathrm{fr}}^{\mathrm{G}^{\prime}}+\stackrel{\nabla}{\boldsymbol{\sigma}}_{\mathrm{vi}}^{\mathrm{G}^{\prime}}, \\
& \stackrel{\nabla}{\mathrm{fr}}^{\mathrm{G}^{\prime}}=\boldsymbol{c}_{\mathrm{fr}}^{\mathrm{G}^{\prime}}\left(\boldsymbol{\sigma}_{\mathrm{fr}}^{\mathrm{G}^{\prime}}, n^{\mathrm{G}}, \boldsymbol{h}^{\mathrm{G}}\right):\left(\left\langle\boldsymbol{d}_{\mathrm{G}}\right\rangle+\frac{1}{3} \dot{p}^{\mathrm{fG}} / K^{\mathrm{sG}} \boldsymbol{I}\right), \\
& \dot{p}^{\mathrm{G}^{\prime}}=-K_{\mathrm{dr}}^{\mathrm{G}}\left(\operatorname{div}\left\langle\boldsymbol{v}_{\mathrm{G}}\right\rangle+\dot{p}^{\mathrm{fG}} / K^{\mathrm{sG}}\right), \\
& K_{\mathrm{dr}}^{\mathrm{G}}=\frac{1}{3} \boldsymbol{I}: \boldsymbol{c}_{\mathrm{fr}}^{\mathrm{G}^{\prime}}: \boldsymbol{I}, \\
& \vec{\nabla}_{\mathrm{vi}}^{\mathrm{G}^{\prime}}=2 \mu^{\mathrm{G}^{\prime}}\left\langle\boldsymbol{\nabla}_{\mathrm{G}}\right\rangle_{\mathrm{dev}},
\end{aligned}
$$

d) interstitial fluid:

$$
\begin{aligned}
\boldsymbol{\sigma}^{\mathrm{fG}} & =-p^{\mathrm{fG}} \boldsymbol{I}+2 \mu^{\mathrm{fG}}\left\langle\boldsymbol{d}_{\mathrm{G}}\right\rangle_{\mathrm{dev}}, \\
\dot{p}^{\mathrm{fG}} & =-\zeta^{\mathrm{G}}\left(\frac{\zeta^{\mathrm{G}}-n^{\mathrm{G}}}{K^{\mathrm{sG}}}+\frac{n^{\mathrm{G}}}{K^{\mathrm{fG}}}\right)^{-1} \operatorname{div}\left\langle\boldsymbol{v}_{\mathrm{G}}\right\rangle,
\end{aligned}
$$


with

$$
\operatorname{div}\left\langle\boldsymbol{v}_{k}\right\rangle=\operatorname{div}\langle\boldsymbol{v}\rangle+\frac{\dot{f}^{k}}{f^{k}} \quad \text { resp. } \quad\left\langle\boldsymbol{d}_{k}\right\rangle=\langle\boldsymbol{d}\rangle+\frac{\dot{f}^{k}}{3 f^{k}} \boldsymbol{I},
$$

by the evolution equations for the material volume fractions,

$$
\dot{f}^{k}=f^{k}\left(\frac{\langle K\rangle}{K^{k}}-1\right) \operatorname{div}\langle\boldsymbol{v}\rangle
$$

and for the porosity of granular material,

$$
\dot{n}^{\mathrm{G}}=\left(1-n^{\mathrm{G}}\right)\left(\frac{\dot{p}^{\mathrm{sG}}}{K^{\mathrm{sG}}}+\operatorname{div}\left\langle\boldsymbol{v}_{\mathrm{G}}\right\rangle\right),
$$

with

$$
\dot{p}^{\mathrm{sG}}=-\frac{K_{\mathrm{dr}}^{\mathrm{G}}}{1-n^{\mathrm{G}}} \operatorname{div}\left\langle\boldsymbol{v}_{\mathrm{G}}\right\rangle+\frac{\zeta^{\mathrm{G}}-n^{\mathrm{G}}}{1-n^{\mathrm{G}}} \dot{p}^{\mathrm{fG}},
$$

and by the compression models for each constituent,

$$
\begin{aligned}
& \dot{\rho}^{\mathrm{S}}=\frac{\rho^{\mathrm{S}}}{K^{\mathrm{S}}}\langle\dot{p}\rangle, \quad \dot{\rho}^{\mathrm{F}}=\frac{\rho^{\mathrm{F}}}{K^{\mathrm{F}}}\langle\dot{p}\rangle, \\
& \dot{\rho}^{\mathrm{sG}}=\frac{\rho^{\mathrm{sG}}}{K^{\mathrm{sG}}} \dot{p}^{\mathrm{sG}}, \quad \dot{\rho}^{\mathrm{fG}}=\frac{\rho^{\mathrm{fG}}}{K^{\mathrm{fG}}} \dot{p}^{\mathrm{fG}} .
\end{aligned}
$$

\section{Discussion and numerical implementation}

\subsection{Features of the model}

The averaged resp. macroscopic balance equations derived in Sect. 2 describe, in the most general case, the non-reactive isothermal flow of an immiscible mixture of $M$ materials consisting of $N$ phases. Because the equations include separate physical quantities (stress, mass density, etc.) for each constituent, separate terms representing the interaction (mass and momentum exchange) between the constituents, as well as the volume fractions of each material and each phase of a material, the macroscopic balance equations can explicitly represent diverse compositions or evolving configurations of 
multi-material flow. The mechanical behavior of the mixture results from the mechanical behavior of its individual constituents and their interactions.

Restrictions have been imposed upon the general equations in Sects. 3 and 4 to describe a geomechanical multi-material example flow of bulk solid, bulk fluid, and fluid-saturated granular material. The assumption of pressure equilibrium between bulk materials, $(40)_{1}$, is adequate because the speed of sound in each material is large compared with velocities in the problems of interest. In other words, equilibration is infinitely fast such that pressure is continuous across a material interface. Pressure disequilibration, on the other hand, would be associated with highly-dynamic compaction, acoustic effects, or other processes not considered here.

The assumption $(40)_{2}$ was very useful with regard to the topological closure of the developed mixture model. However, it is not generally a reasonable one because equilibration of velocity differences is driven by drag forces on material interfaces (momentum transfer). As a consequence of the assumption $(40)_{2}$, the materials are fully bonded without a contact mechanism, and shear resistance is accounted for only by the constitutive equation of the materials next to the interface.

For the granular material we have assumed undrained conditions, as formalized through (35). In contrast to the pressure distribution between the materials which is assumed homogeneous, the pressures in the solid and fluid phases of granular material generally differ. The pressure difference or excess pressure, $(26)_{2}$, results from quasi-static grain contacts in a grain skeleton and is responsible for the frictional behavior of Mohr-Coulomb type; cf. Sect. 3. Pressures might equilibrate, for example, in a fully liquified/fluidized state 
$\left(p^{\mathrm{G}^{\prime}}=0\right)$ with no intergranular contacts and where each grain is completely surrounded by fluid.

The bulk modulus $K^{\mathrm{G}}$ given by (39) holds for arbitrary compositions of saturated grain-fluid mixtures with compressible constituents and homogeneous velocity, (35). Particular cases included are: (i) solid without any pores $\left(n^{\mathrm{G}}=0, K_{\mathrm{dr}}^{\mathrm{G}}=K^{\mathrm{sG}}, \zeta^{\mathrm{G}}=0\right)$, for which $K^{\mathrm{G}}=K^{\mathrm{sG}} \equiv K^{\mathrm{S}}$; (ii) fluid without any solid content $\left(n^{\mathrm{G}}=1, K_{\mathrm{dr}}^{\mathrm{G}}=0, \zeta^{\mathrm{G}}=1\right)$, for which $K^{\mathrm{G}}=K^{\mathrm{fG}} \equiv K^{\mathrm{F}}$; (iii) dry granular material $\left(0<n^{\mathrm{G}}<1, K^{\mathrm{fG}} \approx 0\right)$, for which $K^{\mathrm{G}}=K_{\mathrm{dr}}^{\mathrm{G}}$; (iv) uniform suspension of zero friction $\left(K_{\mathrm{dr}}^{\mathrm{G}}=0, \zeta^{\mathrm{G}}=1\right)$, for which $p^{\mathrm{sG}}=p^{\mathrm{fG}}$ and $K^{\mathrm{G}}=\left(\left(1-n^{\mathrm{G}}\right) / K^{\mathrm{sG}}+n^{\mathrm{G}} / K^{\mathrm{fG}}\right)^{-1}$, known as Wood's equation [99, p. 327].

The assumptions of zero mass and momentum transfer, (41), comply with the assumption of no phase change or chemical reaction at interfaces and zero relative material velocity $(40)_{2}$, respectively. Concerning granular material, the assumption $\boldsymbol{\Gamma}^{\alpha k}=\mathbf{0}$ has at least two interpretations [100, 77]: either the granular material is dry $\left(\rho^{\mathrm{fG}}=0\right)$ or locally undrained. In the second case no consolidation effects take place, and (35) holds.

Despite the restrictions above, no limitations whatsoever exist with regard to the miscibility of the mesoscale continua on the macroscale, in which the materials maintain their original properties, including the granular material. The underlying averaged description of the multi-material flow likewise captures separated mixtures (cf. Fig. 2; analogy: oil on water) as well as disperse mixtures (e.g. granular material containing secondary voids; analogy: emulsion of oil and water).

The derived volume fraction evolution equation, (55) resp. (75), naturally 
provides for a mixture compression model and a void collapse mechanism: the material with the smallest bulk modulus contributes most to the total volume change. This feature is of particular importance in cases where the compressibilities of the materials are widely different. Because of (53) and the basic constraint $\sum_{k} f^{k}=1$, summation of (55) over the materials in the mixture results in (52). Hence, the topological closure relation also satisfies the constraint $\sum_{k} \dot{f}^{k}=0$.

Finally, we remark that the decomposition of stress (23) in conjunction with a compression model is a fundamental concept of our theory in order to treat all materials that might be present in geomechanical multi-material flow, either compressible or incompressible, in a unified fashion. This also becomes apparent from the mixing rules (59)-(63) as well as from the constitutive equations (65)-(73), where we provide formulas that determine the associated bulk modulus from a general material tangent tensor.

In case of single-phase incompressible material (i.e. $f^{k} \equiv 1, n^{k} \in\{0,1\}$ ) the bulk modulus in $(58)_{2}$ becomes infinite, so that pressure plays the role of a Lagrange multiplier ensuring zero divergence of the velocity field. In case of a mixture containing both compressible and incompressible bulk materials, the pressure of the mixture is calculated from $(58)_{2}$. The volume fraction evolution equation (75) maintains pressure equilibrium, $(40)_{1}$, and enforces zero relative volume change of incompressible materials:

$$
-\frac{\dot{\rho}^{k}}{\rho^{k}}=\operatorname{div}\langle\boldsymbol{v}\rangle+\frac{\dot{f}^{k}}{f^{k}}=\frac{\langle K\rangle}{K^{k}} \operatorname{div}\langle\boldsymbol{v}\rangle=0
$$

for $K^{k} \rightarrow \infty$. 


\subsection{Numerical example: isotropic compression}

To give an impression of how the models performs, we consider a mixture of steel (bulk solid), air (bulk fluid), and dry sand subjected to quasi-static isotropic compression. Under these conditions only the second equation of (58) needs to be integrated in time; a simple first-order explicit scheme has been implemented for this purpose. The initial volume fractions are $f_{0}^{\mathrm{S}}=0.4$,

$f_{0}^{\mathrm{F}}=0.2$, and $f_{0}^{\mathrm{G}}=0.4$, respectively, and the initial porosity of the sand is $n_{0}^{\mathrm{G}}=0.4$. The sand is chosen to be uniform and fine-grained, with an angle of internal friction $\phi=32^{\circ}$. The initial pressure of all constituents is the atmospheric pressure at sea level, $p_{\text {atm }}=101.0 \mathrm{kPa}$.

Steel under isotropic compression can be regarded hypoelastic, with constant bulk modulus $K^{\mathrm{S}}=1.6 \times 10^{8} \mathrm{kPa}$. Moreover, we assume for simplicity that the compressibility of the bulk air and the air trapped in the sand pores does not change with pressure, hence $K^{\mathrm{F}}=K^{\mathrm{fG}}=p_{\mathrm{atm}}=$ const.

We employ Janbu's power law [101] in order to efficiently model nonlinear stiffness of the granular material:

$$
E_{\mathrm{s}} \stackrel{\text { def }}{=} C\left(\frac{-\sigma^{\prime}}{p_{\text {atm }}}\right)^{a} p_{\text {atm }} .
$$

$E_{\mathrm{s}}$ is the confined stiffness of the bulk granular material as measured in the socalled oedometer test, achieving one-dimensional compression at zero lateral displacements. $\sigma^{\prime}$ is the effective stress component in loading direction and $C, a$ are constants. For uniform fine sand, the values $C=300$ and $a=0.6$ are reasonable. Jaky's formula [102] provides a relation between $\sigma^{\prime}$ in the oedometer test and the mean effective stress, leading to

$$
\sigma^{\prime}=-\frac{3\langle p\rangle}{1+2(1-\sin \phi)}
$$




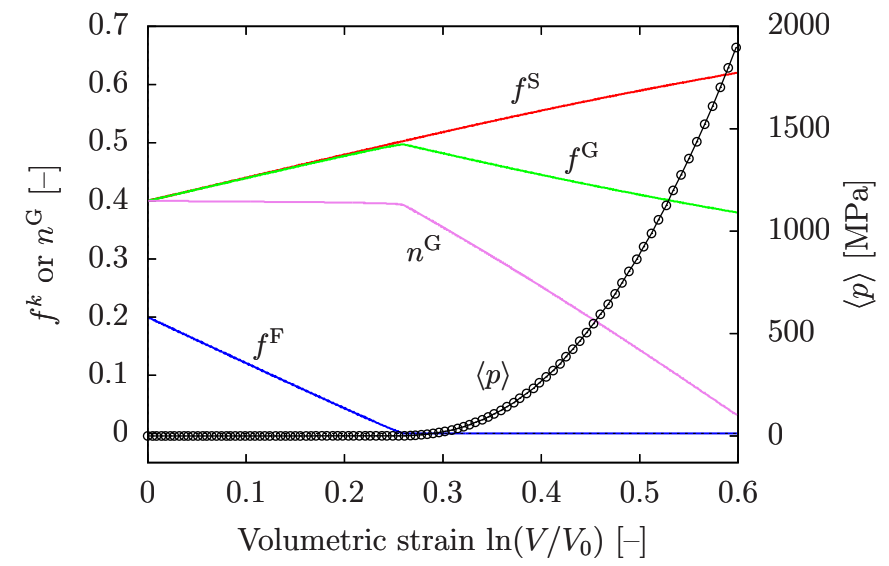

Figure 3: Example application of the homogeneous equilibrium model: mixture of steel (bulk solid), air (bulk fluid), and dry sand under quasi-static isotropic compression. Evolution of the volume fractions, sand porosity, and mixture pressure.

Elasticity theory finally relates $E_{\mathrm{s}}$ with $K_{\mathrm{dr}}^{\mathrm{G}}$, the drained bulk modulus of the granular material:

$$
K_{\mathrm{dr}}^{\mathrm{G}}=E_{\mathrm{s}} \frac{1+\nu}{3(1-\nu)} .
$$

Poisson's ratio is estimated from $\nu=(1-\sin \phi) /(2-\sin \phi)$.

The results plotted in Fig. 3 are reasonable. Under isotropic compression of the mixture the bulk air is compressed first, which does not significantly change the pressure of the materials and the porosity of the granular material. The increase in bulk solid and granular material volume fractions is approximately the same due to (75) and the fact that mixture compressibility is relatively high at this stage of compression. Once the bulk air has been completely compressed, pressure increases exponentially because of the power law (80) and compaction of the granular material, which in turn gives more weight to the relatively high bulk modulus of steel in the calculation of 
the mixture bulk modulus, (62).

\subsection{Full-scale numerical implementation}

In order to analyze real flow situations using the model summarized in Sect. 4.4, the spatial distribution and constitutive closure relations for each material have to be specified, and then the set of equations (58) can be solved in conjunction with (59)-(78) subject to the initial conditions and boundary conditions defining the problem. However, the complexity and nonlinearity of the model makes it impossible to derive analytical solutions for the problems of interest. Therefore, the entire model has to be implemented into a computer code and solved numerically. One approach to achieve this is to employ the arbitrary Lagrangian-Eulerian (ALE) methodology outlined in what follows; see also Sect. 1. Details can be found in $[29,30,103]$, and different numerical approaches for multi-material flows are reviewed in $[6,7,8]$.

The ALE formulation $[104,6,26]$ introduces a reference domain which may move in space at an arbitrary velocity $\boldsymbol{w}$ generally different from the material velocity $\boldsymbol{v}$. For $\boldsymbol{w}=\boldsymbol{v}$ the ALE formulation reduces to the Lagrangian formulation, and for $\boldsymbol{w}=\mathbf{0}$ the Eulerian formulation is obtained. The Eulerian formulation of the set of equations (58) can be written in compact form $[26,30]$

$$
\frac{\partial q}{\partial t}+\operatorname{div} F=S
$$

where $q \in\{\rho \boldsymbol{v}, p\}$ is the quantity under consideration, $F$ is the convective flux of $q$, and $S$ is the source term; angle brackets have been dropped for notational brevity. Instead of solving (83) monolithically, almost all ALE 


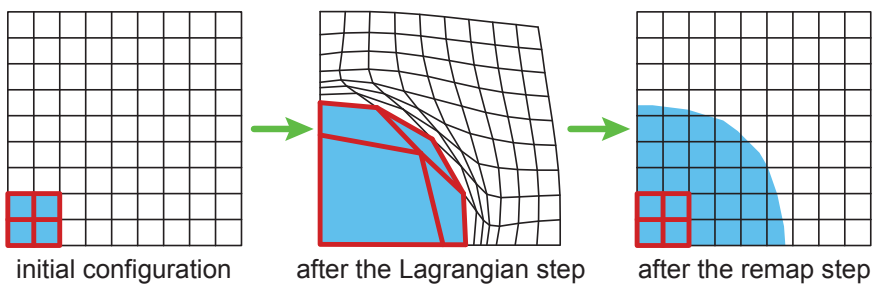

Figure 4: Schematic diagram of a calculational cycle in a typical multi-material ALE method. The blue area indicates a material zone whose initial configuration is assigned to a group of computational cells highlighted in red.

methods apply a Lagrange-remap strategy [6, 26, 27]. Conceptually, (83) is split into two sets of equations which are solved sequentially:

$$
\frac{\partial q}{\partial t}=S \quad \text { and } \quad \frac{\partial q}{\partial t}+\operatorname{div} F=0 .
$$

Fig. 4 illustrates the concept.

The first set of equations in (84) includes the sources but ignores the convective term (i.e. $\boldsymbol{v}=\boldsymbol{w}$ ). Therefore, it is equivalent to the Lagrangian formulation (58) and can be solved e.g. with nonlinear finite element methods accounting for large material deformations $[77,49,105]$. The rate constitutive equations representing path-dependent material behavior, (65) and (68), are conveniently integrated in the Lagrangian step in a frame of reference corotating with the material $[75,26]$.

The velocity-pressure mixed form of momentum balance represented by (58) is a consequence of stress decomposition (23) and suitable for both compressible and incompressible materials [106]; see also discussion in Sect. 5. However, highly compressible materials such as void may cause numerical difficulties on the local and global solution levels if a stiffness matrix has to be inverted. One way to prevent this is to eliminate the nodes that are 
surrounded by zero stiffness cells from the system of equations and to scale the incremental displacements over a time step at material boundaries [107].

The second set of equations in (84) is associated with the remap step. During the remap the distortion of the computational mesh is reduced and then the solution variables are transferred onto the modified mesh. If the ALE method is run in the Eulerian limit $(\boldsymbol{w}=\mathbf{0})$, the mesh nodes are

relocated to their original positions. Since mesh topology does not change, the solution variables can be remapped by using CFD advection algorithms based on finite difference or finite volume approximations $[6,26]$. Time is advanced only during the Lagrangian step, whereas the spatial distributions of the solution variables are fixed during the remap step.

In the multi-material ALE resp. Eulerian approach depicted in Fig. 4, interfaces and free surfaces are not necessarily aligned with cell boundaries but may flow through the mesh $[9,10,11,12,13,14,29,30]$. The heterogeneous mixture in those multi-material cells cut by one or more interfaces is represented as an effective single-phase material using the mixing rules (59)-(63). The interfaces can be tracked by different techniques [108, 109, 110]. Volume of fluid (VOF) methods [111, 9], for example, use the volume fraction data in the cells as it naturally carries information based on which material interfaces can be reconstructed - note that the presence of a $k$-material interface in a bounded domain is characterized by a fractional volume of $0<f^{k}<1$.

\section{Conclusions and outlook}

A theoretical framework based on hybrid mixture theory has been developed in order to model the large-scale motions and interactions of immiscible 
compressible constituents in geomechanical multi-material flow. Macroscopic balance principles have been derived from the corresponding balance principles on the microscale by employing local volume averaging as the filtering technique. The averaging procedure involves a product of two indicator functions through which it is basically an extension of the classical averaging procedure to a hierarchy of three spatial scales (micro, meso, macro). The mesoscale has been introduced as an intermediate scale beyond which even heterogeneous materials in the flow can be represented as continua (bulk materials). In contrast to common two-scale theories, the proposed three-scale approach is able to incorporate both the evolution of bulk material interfaces as well as the two-phase phenomena associated with fluid-saturated granular material.

As in other hybrid mixture theories, the essential closure relations for interfacial transfer, material behavior, and topological closure have been specified with respect to the macroscale. Each constituent has been assumed compressible. The hybrid mixture approach allows for the use of phenomenological constitutive models describing granular material response for the full frictional-collisional flow regime. Accordingly, the apparent stress tensors in the dense grain-fluid mixture have been split into rate-independent and rate-dependent parts. Topological closure has been achieved by the fundamental assumption of homogenous distributions of pressure and velocity, and has finally resulted in a homogenous equilibrium mixture model governing geomechanical multi-material flow.

Future work will focus on the application of the homogenous equilibrium model to specific geomechanical flow situations. For this purpose the author 
and his colleagues currently develop a full-scale multi-material ALE method that numerically solves the system of equations (58); cf. [28, 29, 30]. By recalling the basic solution procedure outlined in this paper, we emphasize that the developed mixture model is particularly suitable for such an endeavor because of two facts: (i) the mixture is effectively modeled as a single component material with homogeneous degrees of freedom at all locations, and (ii) the material volume fraction is a solution variable based on which material interfaces can be reconstructed.

Further research is motivated by the assumptions and restrictions associated with the present paper. For example, the incorporation of mass and momentum transfer between the constituents would be of great practical relevance because geomechanical problems are often driven by local drainage and consolidation phenomena as well as by contact constraints.

\section{Acknowledgements}

The presented work was carried out under the financial support from the German Research Foundation (DFG; Grants SA 310/26-1 and SA 310/26-2) as part of the DFG Research Unit FOR 1136, which is gratefully acknowledged. I want to thank my colleagues in this research unit for several fruitful discussions about my work. I am particularly grateful to my advisors and leaders of the research project, Prof. Stavros A. Savidis and Prof. Frank Rackwitz, for giving me the opportunity to work on this interesting topic and for their continuous support. The helpful comments from the anonymous reviewers and from the co-editor of the journal, Prof. Fernando Pinho, are greatly appreciated. 


\section{Appendix A. Hypoplastic model for frictional granular material behavior}

This appendix summarizes a comprehensive hypoplastic constitutive equation for the frictional behavior of granular material that has been proposed in [87, 88] and extended in [89]. Hypoplasticity, in contrast to elasto-plasticity, is a framework which neither distinguishes between elastic and plastic rates of strains nor defines a yield surface or plastic potential [91]. Instead the constitutive equation for the stress rate is a closed-form expression nonlinear in the rate of deformation.

In order to simplify the notation of the main text we drop angle brackets as well as sub- and superscripts in what follows, and write (49) resp. (68) in the condensed, equivalent form

$$
\stackrel{\nabla}{\stackrel{\text { def }}{=}} \boldsymbol{c}(\boldsymbol{\sigma}, n, \boldsymbol{h}): \boldsymbol{d} .
$$

The hypoplastic constitutive equation considered here describes the frictional stress-strain behavior of isotropic granular materials under a wide range of stress states and states of relative density (resp. porosity) by using only a single set of material constants. Moreover, it accounts for the density-dependence of dilatancy and strength (peak friction) and explicitly models asymptotic states, like the critical state (cf. Sect. 3.1). Liquefaction and other phenomena associated with undrained or constant-volume conditions are well reproduced.

The effective Cauchy stress, $\boldsymbol{\sigma}$, and the void ratio, $e$, are considered as the material state variables. Since the void ratio is related to the porosity 
by $e=n /(1-n)$, their rates are related by

$$
\dot{e}=\frac{\dot{n}}{1-n}\left(1+\frac{n}{1-n}\right)
$$

with $\dot{n}$ given by (76). The hypoplastic evolution of the effective stress is modeled by

$$
\stackrel{\nabla}{\stackrel{\text { def }}{=}} \boldsymbol{c}(\boldsymbol{\sigma}, e, \boldsymbol{d}): \boldsymbol{d} \stackrel{\text { def }}{=} \boldsymbol{L}: \boldsymbol{d}+\boldsymbol{N}\|\boldsymbol{d}\|
$$

where

$$
\begin{aligned}
& \boldsymbol{L}(\boldsymbol{\sigma}, e) \stackrel{\text { def }}{=} \frac{f_{\mathrm{b}} f_{\mathrm{e}}}{\operatorname{tr}\left(\tilde{\boldsymbol{\sigma}}^{2}\right)}\left(F^{2} \mathbf{1}+a^{2} \tilde{\boldsymbol{\sigma}} \otimes \tilde{\boldsymbol{\sigma}}\right), \\
& \boldsymbol{N}(\boldsymbol{\sigma}, e) \stackrel{\text { def }}{=} f_{\mathrm{b}} f_{\mathrm{e}} f_{\mathrm{d}} \frac{a F}{\operatorname{tr}\left(\tilde{\boldsymbol{\sigma}}^{2}\right)}\left(\tilde{\boldsymbol{\sigma}}+\tilde{\boldsymbol{\sigma}}_{\mathrm{dev}}\right), \\
& \mathbf{1} \stackrel{\text { def }}{=} \frac{1}{2}(\boldsymbol{I} \otimes \boldsymbol{I}+\boldsymbol{I} \otimes \boldsymbol{I}), \\
& F \stackrel{\text { def }}{=} \sqrt{\frac{1}{8} \tan ^{2} \psi+\frac{2-\tan ^{2} \psi}{2+\sqrt{2} \tan \psi \cos 3 \theta}}-\frac{1}{2 \sqrt{2}} \tan \psi \text {, } \\
& a \stackrel{\text { def }}{=} \frac{\sqrt{3}\left(3-\sin \phi_{\mathrm{c}}\right)}{2 \sqrt{2} \sin \phi_{\mathrm{c}}} \\
& \tan \psi \stackrel{\text { def }}{=} \sqrt{3}\left\|\tilde{\boldsymbol{\sigma}}_{\text {dev }}\right\| \text {, } \\
& \cos 3 \theta \stackrel{\text { def }}{=}-\sqrt{6} \frac{\operatorname{tr}\left(\tilde{\boldsymbol{\sigma}}_{\mathrm{dev}}^{3}\right)}{\left(\operatorname{tr}\left(\tilde{\boldsymbol{\sigma}}_{\mathrm{dev}}^{2}\right)\right)^{\frac{3}{2}}}, \\
& \tilde{\boldsymbol{\sigma}} \stackrel{\text { def }}{=}-\frac{\boldsymbol{\sigma}}{3 p}, \quad p \stackrel{\text { def }}{=}-\frac{1}{3} \operatorname{tr} \boldsymbol{\sigma}, \quad \tilde{\boldsymbol{\sigma}}_{\mathrm{dev}} \stackrel{\text { def }}{=} \tilde{\boldsymbol{\sigma}}-\frac{1}{3} \boldsymbol{I}, \\
& f_{\mathrm{e}}(\boldsymbol{\sigma}, e) \stackrel{\text { def }}{=}\left(\frac{e_{\mathrm{c}}}{e}\right)^{\beta}, \\
& f_{\mathrm{d}}(\boldsymbol{\sigma}, e) \stackrel{\text { def }}{=}\left(\frac{e-e_{\mathrm{d}}}{e_{\mathrm{c}}-e_{\mathrm{d}}}\right)^{\alpha}, \\
& f_{\mathrm{b}}(\boldsymbol{\sigma}) \stackrel{\text { def }}{=} \frac{\frac{h_{\mathrm{s}}}{n}\left(\frac{1+e_{\mathrm{i}}}{e_{\mathrm{i}}}\right)\left(\frac{e_{\mathrm{i} 0}}{e_{\mathrm{c} 0}}\right)^{\beta}\left(\frac{3 p}{h_{\mathrm{s}}}\right)^{1-n}}{3+a^{2}-\sqrt{3} a\left(\frac{e_{\mathrm{i} 0}-e_{\mathrm{d} 0}}{e_{\mathrm{c} 0}-e_{\mathrm{d} 0}}\right)^{\alpha}},
\end{aligned}
$$


with the characteristic void ratios $e_{\mathrm{i}}(p), e_{\mathrm{c}}(p)$, and $e_{\mathrm{d}}(p)$ being obtained through Bauer's formula [88]

$$
\frac{e_{\mathrm{i}}}{e_{\mathrm{i} 0}} \stackrel{\text { def }}{=} \frac{e_{\mathrm{c}}}{e_{\mathrm{c} 0}} \stackrel{\text { def }}{=} \frac{e_{\mathrm{d}}}{e_{\mathrm{d} 0}} \stackrel{\text { def }}{=} \exp \left(-\left(\frac{3 p}{h_{\mathrm{s}}}\right)^{n}\right) .
$$

The hypoplastic model contains eight material constants: $\phi_{\mathrm{c}}$ is the friction angle at critical state $\left(\right.$ in $\left.^{\circ}\right), h_{\mathrm{s}}$ is a reference pressure called granulate hardness (in $\mathrm{Pa}), e_{\mathrm{d} 0}$ and $e_{\mathrm{i} 0}$ are the minimum and maximum void ratio at zero pressure, respectively, $e_{\mathrm{c} 0}$ is the void ratio at critical state at zero pressure, and $n, \alpha, \beta$ are exponents. The determination of the material constants from experimental laboratory test is described in [89, 90, 112], and sets of constants for some granular materials are listed in $[112,26]$.

\section{Nomenclature}

\section{Operators and special notations}

$\cdot,:, \quad$ single, double contraction, tensor product

$\cup, \cap, \backslash \quad$ union, intersection, and difference of sets

$\langle\cdot\rangle \quad$ spatial average

$(\cdot)^{\alpha k} \quad$ material time derivative of $\alpha k$-field along $\boldsymbol{v}^{\alpha k}$

$(\cdot)^{[\alpha k]} \quad$ limit value at $\alpha k$-boundary 


$\begin{array}{ll}(\cdot) & \text { Zaremba-Jaumann rate } \\ \|\cdot\| & \text { Frobenius norm } \\ \nabla(\cdot) & \text { covariant derivative, gradient } \\ \operatorname{div}(\cdot) & \text { divergence } \\ \operatorname{tr}(\cdot) & \text { trace } \\ \text { Superscripts } & \text { and subscripts } \\ \text { dev } & \text { deviator of a second-order tensor } \\ \text { dr } & \text { drained } \\ \text { e } & \text { elastic } \\ \mathrm{f}, \mathrm{fG}, \mathrm{f} k & \text { fluid phase, in granular material, in } k \text {-material } \\ \mathrm{fr} & \text { frictional (rate-independent) contribution } \\ \mathrm{F} & \text { bulk fluid; F } \equiv \text { fF } \\ \mathrm{G} & \text { fluid-saturated granular material } \\ \mathrm{G} & \text { related to effective stress in granular material } \\ k & \text { plastic } \\ \mathrm{f} & \end{array}$




\begin{tabular}{|c|c|}
\hline $\mathrm{s}, \mathrm{sG}, \mathrm{sk}$ & solid phase, in granular material, in $k$-material \\
\hline $\mathrm{S}$ & bulk solid; $\mathrm{S} \equiv \mathrm{sS}$ \\
\hline $\mathrm{T}$ & transpose of a tensor \\
\hline uj & unjacketed \\
\hline vi & viscous (rate-dependent) contribution \\
\hline$\alpha$ & $\alpha$-phase; $\alpha \in\{\mathrm{s}, \mathrm{f}\}=\{1, \ldots, N\}$ \\
\hline$\alpha k$ & $\alpha$-phase in $k$-material; $\alpha k \in\{\mathrm{S}, \mathrm{F}, \mathrm{sG}, \mathrm{fG}\}$ \\
\hline \multicolumn{2}{|c|}{ Latin symbols } \\
\hline $\boldsymbol{b}, \boldsymbol{b}^{\alpha k},\langle\boldsymbol{b}\rangle$ & body force per unit mass \\
\hline$c^{\mathrm{S}}, \boldsymbol{c}_{\mathrm{fr}}^{\mathrm{G}^{\prime}}$ & fourth-order material tangent tensor \\
\hline $\boldsymbol{d}^{k}, \boldsymbol{d}^{\alpha k},\langle\boldsymbol{d}$ & spatial rate of deformation \\
\hline$\left\langle\boldsymbol{d}_{k}\right\rangle$ & effective rate of deformation of $k$-material \\
\hline $\mathrm{d} v$ & volume density \\
\hline $\mathcal{D}$ & modeling domain in Euclidian space $\mathbb{R}^{3}$ \\
\hline$e^{\mathrm{G}}$ & void ratio \\
\hline$f^{k}$ & $k$-material volume fractions \\
\hline
\end{tabular}


G

$\boldsymbol{h}^{\mathrm{S}}, \boldsymbol{h}^{\mathrm{G}^{\prime}}$

I

$J_{2}$

$K^{\alpha k}, K^{k},\langle K\rangle$

M

$\mathcal{M}^{k}$

$n^{k}, n^{\mathrm{G}}$

$\boldsymbol{n}_{\mathrm{I}}^{\alpha k}$

$N$

$p^{\alpha k}, p^{k},\langle p\rangle$

$p^{\mathrm{G}^{\prime}}$

$\mathcal{P}^{\alpha}$

$q, q^{\alpha k}$

$\boldsymbol{s}^{k}, \boldsymbol{s}^{\alpha k},\langle\boldsymbol{s}\rangle$

$\boldsymbol{v}, \boldsymbol{v}^{k}, \boldsymbol{v}^{\alpha k},\langle\boldsymbol{v}\rangle$ shear modulus

set of material state variables

second-order unit tensor

von Mises stress invariant

bulk modulus

number of materials in the mixture

$k$-material domain in $\mathcal{D}$

fluid fraction, porosity

outward normals on interface

number of phases in the mixture

pressure

mean effective stress

$\alpha$-phase domain in $\mathcal{D}$

generic spatial field

extra stress

spatial velocity 


\begin{tabular}{|c|c|}
\hline $\boldsymbol{v}_{\mathrm{I}}, \boldsymbol{v}_{\mathrm{I}}^{\mathrm{m}}$ & interface velocity \\
\hline$V, V^{k}, V^{\alpha k}$ & volume measures of $\mathcal{V}, \mathcal{V}^{k}, \mathcal{V}^{\alpha k}$ \\
\hline $\mathcal{V}$ & representative volume element (RVE) \\
\hline $\mathcal{V}^{k}, \mathcal{V}^{\alpha k}$ & portions of $k, \alpha k$ in $\mathcal{V}$ \\
\hline$x$ & points in $\mathcal{S}$ \\
\hline$y$ & yield condition \\
\hline \multicolumn{2}{|l|}{ Greek symbols } \\
\hline$\Gamma^{\alpha k}$ & rate of momentum supply via $\partial \mathcal{V}^{\alpha k}$ \\
\hline$\zeta^{\mathrm{G}}$ & Biot-Willis coefficient \\
\hline$\Lambda^{\alpha k}$ & rate of mass supply via $\partial \mathcal{V}^{\alpha k}$ \\
\hline$\mu^{\mathrm{fF}}, \mu^{\mathrm{G}^{\prime}}$ & dynamic shear viscosity \\
\hline$\pi^{\alpha k}$ & volume fraction of $\alpha$ with respect to $\mathcal{V}^{k}$ \\
\hline$\rho, \rho^{\alpha k}, \rho^{k},\langle\rho\rangle$ & spatial mass density \\
\hline$\sigma_{1}, \sigma_{2}, \sigma_{3}$ & principal stresses \\
\hline $\boldsymbol{\sigma}, \boldsymbol{\sigma}^{k}, \boldsymbol{\sigma}^{\alpha k},\langle\boldsymbol{\sigma}\rangle$ & Cauchy stress \\
\hline $\boldsymbol{\sigma}^{\mathrm{G}^{\prime}}$ & effective stress \\
\hline
\end{tabular}




$\begin{array}{ll}\tau_{\mathrm{f}} & \text { shear stress at failure } \\ \phi & \text { angle of internal friction } \\ \chi^{k}, \chi^{\alpha}, \chi^{\alpha k} & \text { indicator function } \\ \boldsymbol{\omega} & \text { vorticity tensor }\end{array}$

\section{References}

[1] S. L. Soo, Fluid Dynamics of Multiphase Systems, Blaisdell Publishing Company, Waltham, Massachusetts, 1967.

[2] G. Hetsroni (Ed.), Handbook of Multiphase Systems, Hemisphere Publishing Corporation, 1982.

[3] M. Ishii, T. Hibiki, Thermo-Fluid Dynamics of Two-Phase Flow, 2nd Edition, Springer Science+Business Media, LLC, 2011.

[4] H. B. Stewart, B. Wendroff, Two-phase flow: Models and methods, Journal of Computational Physics 56 (1984) 363-409.

[5] D. A. Drew, S. L. Passman, Theory of Multicomponent Fluids, Springer, New York, 1999.

[6] D. J. Benson, Computational methods in Lagrangian and Eulerian hydrocodes, Computer Methods in Applied Mechanics and Engineering $99(2-3)(1992)$ 235-394.

[7] H. U. Mair, Review: Hydrocodes for structural response to underwater explosions, Shock and Vibration 6 (1999) 81-96. 
[8] E. S. Hertel, A survey of numerical methods for shock physics applications, Report SAND97-1015C, Sandia National Laboratories, USA (1997).

[9] D. L. Youngs, Time-Dependent Multi-Material Flow with Large Fluid Distortion, Numerical Methods for Fluid Dynamics, Academic Press, London, 1982, pp. 273-285.

[10] J. M. McGlaun, S. L. Thompson, M. G. Elrick, CTH: A threedimensional shock wave physics code, International Journal of Impact Engineering 10 (1990) 351-360.

[11] D. J. Benson, A multi-material Eulerian formulation for the efficient solution of impact and penetration problems, Computational Mechanics 15 (1995) 558-571.

[12] J. S. Peery, D. E. Carroll, Multi-material ALE methods in unstructured grids, Computer Methods in Applied Mechanics and Engineering 187 (2000) 591-619.

[13] W. J. Rider, E. Love, M. K. Wong, O. E. Strack, S. V. Petney, D. A. Labreche, Adaptive methods for multi-material ALE hydrodynamics, International Journal for Numerical Methods in Fluids 65 (2011) 13251337.

[14] S. Galera, J. Breil, P.-H. Maire, A 2d unstructured multi-material cellcentered arbitrary Lagrangian-Eulerian (CCALE) scheme using MOF interface reconstruction, Computers \& Fluids 46 (2011) 237-244. 
[15] D. J. Benson, A mixture theory for contact in multi-material Eulerian formulations, Computer Methods in Applied Mechanics and Engineering 140 (1997) 59-86.

[16] P. Colella, H. M. Glaz, R. E. Ferguson, Multifluid algorithms for Eulerian finite difference methods, (unpublished manuscript) (1997).

[17] G. H. Miller, E. G. Puckett, A high-order Godunov method for multiple condensed phases, Journal of Computational Physics 128 (1996) 134164.

[18] J. Locat, H. J. Lee, Submarine landslides: Advances and challenges, Canadian Geotechnical Journal 39 (2002) 193-212.

[19] D. G. Masson, C. B. Harbitz, R. B. Wynn, G. Pedersen, F. Løvholt, Submarine landslides: processes, triggers and hazard prediction, Philosophical Transactions of the Royal Society of London. Series A 364 (2006) 2009-2039.

[20] R. M. Iverson, The physics of debris flows, Reviews of Geophysics 35 (3) (1997) 245-296.

[21] R. M. Iverson, R. P. Denlinger, Flow of variably fluidized granular masses across three-dimensional terrain. 1. coulomb mixture theory, Journal of Geophysical Research 106 (2001) 537-552.

[22] S. P. Pudasaini, K. Hutter, Avalanche Dynamics: Dynamics of Rapid Flows of Dense Granular Avalanche, Springer-Verlag Berlin Heidelberg, 2007. 
[23] H. B. Seed, K. L. Lee, I. M. Idriss, F. I. Makdisi, The slides in the San Fernando dams during the earthquake of February 9, 1971, Journal of the Geotechnical Engineering Division, ASCE 101 (7) (1975) 651-688.

[24] R. B. Seed, K. O. Cetin, R. E. S. Moss, A. M. Kammerer, J. Wu, J. M. Pestana, M. F. Riemer, R. B. Sancio, J. D. Bray, R. E. Kayen, A. Faris, Recent advances in soil liquefaction engineering: a unified and consistent framework, Report EERC 2003-06, Earthquake Engineering Research Center, University of California, Berkeley, USA (2003).

[25] D. J. White, An investigation into the behaviour of pressed-in piles, Ph.D. thesis, University of Cambridge, UK (2002).

[26] D. Aubram, An Arbitrary Lagrangian-Eulerian Method for Penetration into Sand at Finite Deformation, no. 62 in Veröffentlichungen des Grundbauinstitutes der Technischen Universität Berlin, Shaker Verlag, Aachen, 2013, http://dx.doi.org/10.14279/depositonce-3958.

[27] D. Aubram, F. Rackwitz, P. Wriggers, S. A. Savidis, An ALE method for penetration into sand utilizing optimization-based mesh motion, Computers and Geotechnics 65 (2015) 241-249, http://dx.doi.org/ 10.1016/j.compgeo. 2014.12.012.

[28] D. Aubram, F. Rackwitz, S. A. Savidis, Vibro-injection pile installation in sand: Part i-interpretation as multi-material flow, in: T. Triantafyllidis (Ed.), Holistic Simulation of Geotechnical Installation Processes: Numerical and Physical Modelling, Vol. 77 of Lecture Notes in Applied and Computational Mechanics, Springer Inter- 
national Publishing, 2015, pp. 73-102, http://dx.doi.org/10.1007/ 978-3-319-18170-7_5.

[29] S. A. Savidis, D. Aubram, F. Rackwitz, Vibro-injection pile installation in sand: Part ii-numerical and experimental investigation, in: T. Triantafyllidis (Ed.), Holistic Simulation of Geotechnical Installation Processes: Numerical and Physical Modelling, Vol. 77 of Lecture Notes in Applied and Computational Mechanics, Springer International Publishing, 2015, pp. 103-131, http://dx.doi.org/10.1007/ 978-3-319-18170-7_6.

[30] D. Aubram, S. A. Savidis, F. Rackwitz, Theory and numerical modeling of geomechanical multi-material flow, in: T. Triantafyllidis (Ed.), Holistic Simulation of Geotechnical Installation Processes: Benchmarks and Simulations, Vol. 80 of Lecture Notes in Applied and Computational Mechanics, Springer International Publishing, 2016, pp. 187-229, http://dx.doi.org/10.1007/978-3-319-23159-4_10.

[31] M. Hassanizadeh, W. G. Gray, General conservation equations for multi-phase systems: 1. Averaging procedure, Advances in Water Resources 2 (1979) 131-144.

[32] M. Hassanizadeh, W. G. Gray, Mechanics and thermodynamics of multiphase flow in porous media including interphase boundaries, Advances in Water Resources 13 (4) (1990) 169-186.

[33] S. Achanta, J. H. Cushman, M. R. Okos, On multicomponent, multi- 
phase thermomechanics with interfaces, International Journal of Engineering Science 32 (11) (1994) 1717-1738.

[34] L. S. Bennethum, J. H. Cushman, Multiscale, hybrid mixture theory for swelling systems-i: Balance laws, International Journal of Engineering Science 34 (2) (1996) 125-145.

[35] L. S. Bennethum, J. H. Cushman, Multiscale, hybrid mixture theory for swelling systems-ii: Constitutive theory, International Journal of Engineering Science 34 (2) (1996) 147-169.

[36] A. Bedford, D. S. Drumheller, Theories of immiscible and structured mixtures, International Journal of Engineering Science 21 (8) (1983) $863-960$.

[37] J. H. Cushman, L. S. Bennethum, B. X. Hu, A primer on upscaling tools for porous media, Advances in Water Resources 25 (2002) 10431067.

[38] J. A. Bouré, J. M. Delhaye, General Equations and Two-Phase Flow Modeling, Handbook of Multiphase Systems, Hemisphere Publishing Corporation, 1982, Ch. 1.2.

[39] D. A. Drew, Mathematical modeling of two-phase flow, Annual Review of Fluid Mechanics 15 (1983) 261-291.

[40] C. Truesdell, R. A. Toupin, The Classical Field Theories, Vol. III/1 of Encyclopedia of Physics, Springer-Verlag Berlin Göttingen Heidelberg, 1960, pp. 226-793. 
[41] C. Truesdell, W. Noll, The Non-Linear Field Theories of Mechanics, 3rd Edition, Springer-Verlag Berlin Heidelberg New York, 2004.

[42] L. E. Malvern, Introduction to the Mechanics of a Continuous Medium, Prentice Hall, Inc., New Jersey, 1969.

[43] C. Ancey, P. Coussot, P. Evesque, A theoretical framework for granular suspensions in a steady simple shear flow, Journal of Rheology 43 (6) (1999) 1673-1699.

[44] S. B. Savage, Granular flows down rough inclines - review and extension, in: J. T. Jenkins, M. Satake (Eds.), Mechanics of Granular Materials: New Models and Constitutive Equations, Elsevier, Amsterdam, 1983, pp. 261-282.

[45] S. B. Savage, K. Hutter, The motion of a finite mass of granular material down a rough incline, Journal of Fluid Mechanics 199 (1989) $21-24$.

[46] K. G. Anderson, R. Jackson, A comparison of the solutions of some proposed equations of motion of granular materials for fully developed flow down inclined planes, Industrial \& Engineering Chemistry Fundamentals 241 (1992) 145-168.

[47] K. Hutter, K. R. Rajagopal, On flows of granular materials, Continuum Mechanics and Thermodynamics 6 (1994) 81-139.

[48] K. Hutter, B. Svendsen, D. Rickenmann, Debris flow modeling: a review, Continuum Mechanics and Thermodynamics 8 (1996) 1-35. 
[49] O. C. Zienkiewicz, A. H. C. Chan, M. Pastor, B. A. Schrefler, T. Shiomi, Computational Geomechanics with Special Reference to Earthquake Engineering, John Wiley \& Sons, Ltd., 1999.

[50] A. N. Schofield, C. P. Wroth, Critical State Soil Mechanics, McGrawHill, New York, 1968.

[51] W. Wu, H. S. Yu (Eds.), Modern Trends in Geomechanics, Vol. 106 of Springer Proceedings in Physics, Springer-Verlag Berlin Heidelberg, 2006.

[52] J. D. Goddard, Continuum modeling of granular media, Applied Mechanics Reviews 66 (050801).

[53] R. A. Bagnold, Experiments on a gravity-free dispersion of large solid spheres in a newtonian fluid under shear, Proceedings of the Royal Society of London. Series A 225 (1954) 49-63.

[54] P. C. Johnson, R. Jackson, Frictional-collisional constitutive relations for granular materials, with application to plane shearing, Journal of Fluid Mechanics 176 (1987) 67-93.

[55] C. Ancey, P. Evesque, Frictional-collisional regime for granular suspension flows down an inclined channel, Physical Review E 62 (6) (2000) 8349-8360.

[56] C. Ancey, Dry granular flows down an inclined channel: Experimental investigations on the frictional-collisional regime, Physical Review E 65 (011304). 
[57] C. Ancey, Plasticity and geophysical flows: A review, Journal of NonNewtonian Fluid Mechanics 142 (2007) 4-35.

[58] K. Terzaghi, Theoretical Soil Mechanics, John Wiley \& Sons, Inc., New York, 1943.

[59] M. A. Biot, General theory of three-dimensional consolidation, Journal of Applied Physics 12 (1941) 155-164.

[60] M. A. Biot, Theory of elasticity and consolidation for a porous anisotropic solid, Journal of Applied Physics 26 (2) (1955) 182-185.

[61] A. Sawicki, J. Mierczyński, Developments in modeling liquefaction of granular soils, caused by cyclic loads, Applied Mechanics Reviews 59 (2006) 91-106.

[62] P. Coussot, C. Ancey, Rheophysical classification of concentrated suspensions and granular pastes, Physical Review E 59 (4) (1999) 44454457.

[63] Y. Wang, K. Hutter, A constitutive model of multiphase mixtures and its application in shearing flows of saturated solid-fluid mixtures, Granular Matter 1 (1999) 163-181.

[64] L. S. Bennethum, T. Weinstein, Three pressures in porous media, Transport in Porous Media 54 (2004) 1-34.

[65] B. M. Das, Advanced Soil Mechanics, 3rd Edition, Taylor \& Francis, USA, 2008. 
[66] P. V. Lade, R. de Boer, The concept of effective stress for soil, concrete and rock, Géotechnique 47 (1) (1997) 61-78.

[67] L. S. Bennethum, Compressibility moduli for porous materials incorporating volume fraction, Journal of Engineering Mechanics, ASCE 132 (2006) 1205-1214.

[68] L. S. Bennethum, Theory of flow and deformation of swelling porous materials at the macroscale, Computers and Geotechnics 34 (2007) $267-278$.

[69] H. F. Wang, Theory of Linear Poroelasticity with Applications to Geomechanics and Hydrogeology, Princeton University Press, 2000.

[70] M. A. Biot, D. G. Willis, The elastic coefficients of the theory of consolidation, Journal of Applied Mechanics 24 (1957) 594-601.

[71] E. Detournay, A. H.-D. Cheng, Fundamentals of Poroelasticity, Vol. 2: Analysis and Design Methods of Comprehensive Rock Engineering: Principles, Practice and Projects, Pergamon Press, 1993, Ch. 5.

[72] O. C. Zienkiewicz, T. Shiomi, Dynamic behaviour of saturated porous media: The generalized Biot formulation and its numerical solution, International Journal for Numerical and Analytical Methods in Geomechanics 8 (1984) 71-96.

[73] J. A. Bouré, Two-phase flow models: The closure issue, Multiphase Science and Technology 3 (1-4) (1987) 3-30. 
[74] H. A. Jakobsen, Chemical Reactor Modeling: Multiphase Reactive Flows, Springer-Verlag Berlin Heidelberg, 2008.

[75] J. C. Simo, T. J. R. Hughes, Computational Inelasticity, SpringerVerlag Berlin, 1998.

[76] S. Nemat-Nasser, A. Shokooh, On finite plastic flows of compressible materials with internal friction, International Journal of Solids and Structures 16 (1980) 495-514.

[77] R. W. Lewis, B. A. Schrefler, The Finite Element Method in the Static and Dynamic Deformation and Consolidation of Porous Media, 2nd Edition, John Wiley \& Sons, Ltd., Chichester, 1998.

[78] C. Truesdell, Hypo-elasticity, Journal of Rational Mechanics and Analysis 4 (1) (1955) 83-133.

[79] J. C. Simo, K. S. Pister, Remarks on rate constitutive equations for finite deformation problems: Computational implications, Computer Methods in Applied Mechanics and Engineering 46 (1984) 201-215.

[80] S. L. Passman, J. W. Nunziato, P. B. Bailey, K. W. Reed, Shearing motion of a fluid-saturated granular material, Journal of Rheology 30 (1) (1986) 167-192.

[81] Y. Forterre, O. Pouliquen, Flows of dense granular media, Annual Review of Fluid Mechanics 40 (2008) 1-24.

[82] W. F. Chen, D. J. Han, Plasticity for Structural Engineers, SpringerVerlag New York, Inc., 1988. 
[83] M. Pastor, O. C. Zienkiewicz, A. H. C. Chan, Generalized plasticity and the modelling of soil behaviour, International Journal for Numerical and Analytical Methods in Geomechanics 14 (1990) 151-190.

[84] M. T. Manzari, Y. F. Dafalias, A critical state two-surface plasticity model for sands, Géotechnique 47 (2) (1997) 255-272.

[85] M. Taiebat, Y. F. Dafalias, Sanisand: Simple anisotropic sand plasticity model, International Journal for Numerical and Analytical Methods in Geomechanics 32 (2008) 915-948.

[86] X. S. Li, A sand model with state-dependent dilatancy, Géotechnique 52 (3) (2002) 173-186.

[87] G. Gudehus, A comprehensive constitutive equation for granular materials, Soils and Foundations 36 (1) (1996) 1-12.

[88] E. Bauer, Calibration of a comprehensive constitutive equation for granular materials, Soils and Foundations 36 (1) (1996) 13-26.

[89] P.-A. von Wolffersdorff, A hypoplastic relation for granular materials with a predefined limit state surface, Mechanics of Cohesive-Frictional Materials 1 (1996) 251-271.

[90] A. Niemunis, I. Herle, Hypoplastic model for cohesionless soils with elastic strain range, Mechanics of Cohesive-Frictional Materials 2 (1997) 279-299.

[91] D. Kolymbas (Ed.), Introduction to Hypoplasticity, A. A. Balkema, Rotterdam, 2000. 
[92] H. Hwang, K. Hutter, A new kinetic model for rapid granular flow, Continuum Mechanics and Thermodynamics 7 (1995) 357-384.

[93] U. Häussler, J. Eibl, Numerical investigations on discharging silos, Journal of Engineering Mechanics 110 (6) (1984) 957-971.

[94] J. U. Böhrnsen, H. Antes, M. Ostendorf, J. Schwedes, Silo discharge: Measurement and simulation of dynamic behavior in bulk solids, Chemical Engineering \& Technology 27 (2004) 71-76.

[95] S. B. Savage, Gravity flow of cohesionless granular materials in chutes and channels, Journal of Fluid Mechanics 92 (1979) 53-96.

[96] W. Herrmann, Constitutive equation for the dynamic compaction of ductile porous materials, Journal of Applied Physics 40 (6) (1969) 2490-2499.

[97] M. R. Baer, J. W. Nunziato, A two-phase mixture theory for the deflagration-to-detonation transition (DDT) in reactive granular materials, International Journal of Multiphase Flow 12 (6) (1986) 861-889.

[98] D. S. Drumheller, A theory for dynamic compaction of wet porous solids, International Journal of Solids and Structures 23 (2) (1987) 211-237.

[99] A. B. Wood, A Textbook of Sound, The Macmillan Company, New York, 1930.

[100] M. Hassanizadeh, W. G. Gray, General conservation equations for 
multi-phase systems: 3. Constitutive theory for porous media flow, Advances in Water Resources 3 (1980) 25-40.

[101] D. M. Wood, Soil Mechanics: A One-Dimensional Introduction, Cambridge University Press, USA, 2009.

[102] R. F. Craig, Craig's Soil Mechanics, 7th Edition, E \& FN Spon, London, New York, 2007.

[103] D. Aubram, A multi-material Eulerian method for large deformation and free surface flow of geomaterials, (in preparation).

[104] C. W. Hirt, A. A. Amsden, J. L. Cook, An arbitrary LagrangianEulerian computing method for all flow speeds, Journal of Computational Physics 14 (1974) 227-253.

[105] T. Belytschko, W. K. Liu, B. Moran, Nonlinear Finite Elements for Continua and Structures, John Wiley \& Sons, Ltd., 2000.

[106] O. C. Zienkiewicz, R. L. Taylor, The Finite Element Method, 5th Edition, Vol. 1: The Basis, Butterworth-Heinemann, 2000.

[107] D. J. Benson, An implicit multi-material Eulerian formulation, International Journal for Numerical Methods in Engineering 48 (2000) 475499.

[108] J. M. Hyman, Numerical methods for tracking interfaces, Physica D 12 (1984) 396-407. 
[109] R. Scardovelli, S. Zaleski, Direct numerical simulation of free-surface and interfacial flow, Annual Review of Fluid Mechanics 31 (1999) 567603.

[110] D. J. Benson, Volume of fluid interface reconstruction methods for multi-material problems, Applied Mechanics Reviews 55 (2) (2002) 151-165.

[111] C. W. Hirt, B. D. Nichols, Volume of fluid (VOF) method for the dynamics of free boundaries, Journal of Computational Physics 39 (1981) 201-225.

[112] I. Herle, G. Gudehus, Determination of parameters of a hypoplastic constitutive model from properties of grain assemblies, Mechanics of Cohesive-Frictional Materials 4 (1999) 461-486. 